\title{
Aeroelastic Modal Characteristics of Mistuned Blade Assemblies: Mode Localization and Loss of Eigenstructure
}

\author{
Christophe Pierre* \\ University of Michigan, Ann Arbor, Michigan 48109 \\ and \\ Durbha V. Murthy† \\ NASA Lewis Research Center, Cleveland, Ohio 44135
}

\begin{abstract}
An investigation of the effects of small mistuning on the aeroelastic modes of bladed-disk assemblies with aerodynamic coupling between blades is presented. The cornerstone of the approach is the use and development of perturbation methods that exhibit the crucial role of the interblade coupling and yield general findings regarding mistuning effects. It is shown that blade assemblies with weak aerodynamic interblade coupling are highly sensitive to small blade mistuning and that their dynamics are qualitatively altered in the following ways: the regular pattern that characterizes the root locus of the tuned aeroelastic eigenvalues in the complex plane is totally lost; the aeroelastic mode shapes become severely localized to only a few blades of the assembly and lose their constant interblade phase angle feature; curve veering phenomena take place when the eigenvalues are plotted vs a mistuning parameter.
\end{abstract}

\section{Nomenclature}

$\mathbf{A}=$ aerodynamic matrix in physical coordinates

$\tilde{\mathbf{A}}=$ aerodynamic matrix in interblade phase angle coordinates

$\tilde{A}_{j} \quad=$ aerodynamic coefficient for $j$ th interblade phase angle mode

$\mathbf{E}=$ Fourier transformation matrix

$\boldsymbol{e}_{j} \quad=j$ th constant interblade phase angle mode shape

$\mathbf{K}=$ stiffness matrix

$K_{o} \quad=$ nominal generalized blade stiffness

$M \quad=$ generalized mass

$\mathbf{M}=$ mass matrix

$m$ = number of nonrotating blade component modes

$N \quad$ = number of blades

$u=$ aeroelastic mode shape

$u_{i} \quad=i$ th physical coordinate

$\delta \mathbf{K}=$ deviation from tuned stiffness matrix

$\delta K_{i}=$ stiffness mistuning for $i$ th blade

$\delta \lambda \quad$ first-order eigenvalue perturbation

$\delta^{2} \lambda=$ second-order eigenvalue perturbation

$\delta \boldsymbol{u}=$ first-order eigenvector perturbation

$\delta^{2} \boldsymbol{u}=$ second-order eigenvector perturbation

$\epsilon \quad=$ mistuning standard deviation

$\eta_{j} \quad=j$ th interblade phase angle coordinate

$\lambda=$ aeroelastic eigenvalue

$\sigma_{j} \quad=$ interblade phase angle for $j$ th mode

$\omega=$ complex frequency

$\omega_{a}=$ assumed frequency

Received April 1, 1991; presented as Paper 91-1218 at the AIAA/ASME/ASCE/AHS/ASC 32nd Structures, Structural Dynamics, and Materials Conference, Baltimore, MD, April 8-10, 1991; revision received Feb. 10, 1992; accepted for publication Feb. 24, 1992. Copyright (C) 1992 by the American Institute of Aeronautics and Astronautics, Inc. No copyright is asserted in the United States under Title 17, U.S. Code. The U.S. Government has a royalty-free license to exercise all rights under the copyright claimed herein for Governmental purposes. All other rights are reserved by the copyright owner.

*Associate Professor, Department of Mechanical Engineering and Applied Mechanics. Member AIAA.

† Senior Research Associate, MS 23-3, 2100 Brookpark Road; also Senior Research Associate, University of Toledo, Toledo, OH. Senior Member AIAA.

\section{Introduction}

$\mathbf{P}$ ERFECT periodicity, or cyclic symmetry, is a convenient, frequent assumption when the dynamics of bladed disk assemblies is analyzed. A primary reason for taking advantage of cyclic symmetry is that the blade response and excitation can always be expressed in terms of constant interblade phase angle modes that uncouple the equations of motion, thereby reducing the size of the problem to that of one blade. ${ }^{1,2}$ This simplification yields drastic reductions in computational cost. Such ideal regularity, however, holds true only if all the blades are identical and uniformly spaced and if the disk is symmetric. However, periodicity is always disrupted by differences in the blade structural properties and modes of vibration, which result from manufacturing and material tolerances. Cyclic symmetry of the unsteady aerodynamic loading may also be destroyed by a slightly unequal spacing of the blades. This phenomenon, known as mistuning, not only tremendously increases the size and cost of the analysis of blade assemblies such as turbines, compressors, and fans, but may also qualitatively alter their dynamics.

Numerous studies have been conducted in an attempt to understand the effects of mistuning on the dynamics of blade assemblies (see the survey paper by Srinivasan ${ }^{3}$ ). These studies have led to some common conclusions. For example, it has been suggested that, although mistuning has often a beneficial (stabilizing) effect in a flutter situation, ${ }^{4-7}$ it may have an undesirable effect on the forced response through a possibly very large increase in the maximum amplitude experienced by some blades. ${ }^{8}$ It has also been shown that blade mistuning results in the appearance of new peaks in the frequency response. ${ }^{9}$ Besides these general findings, though, there are quantitatively and even qualitatively different results among these studies. For instance, the increase in maximum amplitude due to mistuning, the blade with the largest amplitude, and the effect of mistuning standard deviation on the rotor's largest amplitude were all found to be different by various researchers.

We believe these discrepancies originate from the widely different models and parameter values used in the various studies. This was suggested and substantiated in a series of recent papers. ${ }^{7,10-12}$ These studies showed that the sensitivity to mistuning can vary by several orders of magnitude depending on the strength of the interblade coupling, the excitation frequency, and the number of blades. Specifically, in the weak 
interblade coupling case, small random mistuning drastically alters both the free and forced responses by localizing the vibration to a small geometric region of the structure (or to a few blades) and by increasing severely the amplitudes of some blades-a phenomenon referred to as "mode localization."

These investigations led to a fundamental understanding of mistuning effects. However, except for the pioneering work by Bendiksen $^{7}$ that evidenced the high sensitivity of closely spaced aeroelastic modes to mistuning (although not localization), the localization studies just cited did not include any aerodynamic effects.

In this paper motion-dependent unsteady aerodynamic loads are included in the formulation, leading to an aeroelastic eigenvalue problem that governs the stability of the blade assembly. Our primary objective is to reach general conclusions regarding the effects of small structural mistuning on the aeroelastic mode shapes, eigenvalues, and stability of typical high-mass-ratio turbomachinery blade assemblies. The cornerstone of our approach is the development and use of perturbation schemes that enable us to predict mistuning effects and that yield important physical insights into the dynamics of mistuned assemblies.

To achieve structural mistuning without altering the aerodynamic terms, we consider small, random mistuning of the blade frequencies. Moreover, to highlight the role of aerodynamic coupling on the sensitivity to mistuning, we ignore structural coupling between blades. This enables us to demonstrate the key roles of aerodynamic stiffness and damping. We show that aerodynamic coupling has an effect qualitatively similar to that of structural interblade coupling ${ }^{11}$ (although it has a vastly different quantitative effect). In particular, we find that assemblies with weak aerodynamic interblade coupling (e.g., those for which aerodynamic forces are small compared to structural elastic forces) are highly sensitive to mistuning, and that their dynamics is qualitatively altered. For example, aeroelastic modes become localized and the locus of the eigenvalues loses its structure when small mistuning is introduced.

The paper is organized as follows. Section II presents the structural and aerodynamic models and the formulation of the aeroelastic eigenvalue problem. The dynamics of tuned aeroelastic systems is reviewed in Sec. III. In Sec. IV we discuss the nature of two parameters that are key to our study: the aerodynamic coupling and the structural mistuning. Perturbation schemes that predict and provide insight into mistuning effects are developed in Sec. V. Section VI presents the results of a parametric study of a blade assembly and their interpretation. Section VII concludes the paper.

The primary original contributions of the paper lie 1) in the evidence of new phenomena (e.g., localization of aeroelastic modes and loss of structure of the root locus) occurring in mistuned aeroelastic systems, and 2) in the generality of the mistuning trends and phenomena uncovered by our perturbation approach. We expect our results to be qualitatively valid for typical turbomachinery blade assemblies with stiff blades.

\section{Equations of Aeroelastic Motion}

The structural and aerodynamic models we use in our study are those introduced by Kaza and Kielb. ${ }^{13}$ In this section we briefly describe these models. The reader is referred to Ref. 13 for a full description.

The structure we examine consists of $N$ blades equally spaced on a disk. Each blade is modeled as a straight, slender, twisted, nonuniform elastic beam with a symmetric cross section. The elastic, inertia, and tension axes are taken to be noncoincident, and the effect of warping is considered. The equations of motion of a rotating blade are discretized by expanding the blade deflection in terms of the mode shapes of the associated nonrotating blade. Thus, each blade is effectively modeled by an $m$-degree-of-freedom system, where $m$ is the number of nonrotating blade modes used.
We do not consider blade root flexibility and assume that the blades are unshrouded and rigidly clamped to the disk. Moreover, as in Ref. 13, we take the disk to be rigid. This means that there is no structural coupling between blades in our assembly. Thus, the interblade coupling arises solely from aerodynamic effects. This allows us to highlight the effects of aerodynamic coupling, as those of structural coupling have been studied previously. ${ }^{11}$

The unsteady, motion-dependent aerodynamic forces are calculated by applying two-dimensional, linear, unsteady, cascade aerodynamic theories in a strip fashion for both subsonic $^{14}$ and supersonic ${ }^{15}$ regimes. This results in a (complex) matrix of generalized aerodynamic forces. Motion-independent aerodynamic loads are not considered here, since we restrict our investigation to the aeroelastic eigenvalue problem. Furthermore, to highlight the effect of aerodynamic damping, no structural energy dissipation is included in our model (although linear structural damping could be added easily).

Since each blade is modeled by $m$ natural modes, the motion of a blade is described by $m$ coordinates that are the amplitudes of the motion in those natural modes. We will refer to these modal amplitudes as the blade physical coordinates. Application of component mode analysis to the $N$-blade assembly yields a set of $\mathrm{Nm}$ homogeneous, linear, ordinary differential equations in the blade physical coordinates. We look for motions such that all the blade coordinates oscillate with the same frequency and/or decay or grow at the same rate. This yields the aeroelastic eigenvalue problem:

$$
\left\{-\lambda \mathbf{M}+\mathbf{K}-\mathbf{A}\left(\omega_{a}\right)\right\} \boldsymbol{u}=\mathbf{0}
$$

where $\boldsymbol{u}=\left[u_{11}, \ldots, u_{1 m}, u_{21}, \ldots, u_{2 m}, \ldots, u_{N 1}, \ldots, u_{N m}\right]^{T}$ is the $\mathrm{Nm}$-dimensional complex eigenvector of the blade physical coordinates, where $T$ denotes a transpose; $\mathbf{M}$ and $\mathbf{K}$ are $N m \times N m$ real inertia and stiffness matrices, respectively; $\mathbf{A}$ is the $N m \times N m$ complex aerodynamic matrix, calculated at the assumed frequency $\omega_{a}$; and $\lambda$ is the complex eigenvalue.

The matrices $\mathbf{A}, \mathbf{K}$, and $\mathbf{M}$ consist of $N^{2}$ blocks, each of size $m \times m$. Since there is no structural coupling. between blades, $\mathbf{K}$ is a block-diagonal matrix, where each block is the stiffness matrix of an individual blade (for a nonrotating assembly these blocks themselves are diagonal). The absence of structural coupling also means that $\mathbf{M}$ is block-diagonal.

The aerodynamic matrix $\mathbf{A}$ is fully populated. The off-diagonal blocks provide aerodynamic coupling between the blades, and the off-diagonal elements for each block account for the coupling between the (nonrotating) blade modes. The matrix A depends on the assumed frequency of blade vibration $\omega_{a}$ and on the flow and geometric parameters.

For a tuned system the blades are identical; thus, all matrices in Eq. (1) are block circulant. These block-circulant matrices have special properties that result in a mostly analytical description of the eigensolution of the tuned assembly (this is discussed further in Sec. III). The stiffness matrix of the tuned assembly consists of identical blocks on the diagonal. For example, for a one-component mode per blade representation, $\mathbf{K}$ is proportional to the identity matrix. For a mistuned assembly with arbitrarily different blades, the stiffness matrix consists of distinct blocks and is no longer blockcirculant, but with the assumption of frequency mistuning, the mass and aerodynamic matrices remain block circulant. In fact, the aerodynamic matrix is left unchanged by the introduction of frequency mistuning (if there is no mode shape mistuning).

The solution of the aeroelastic eigenvalue problem [Eq. (1)] dictates the nature and stability of the assembly's motion in an aeroelastic mode. For an eigensolution $(\lambda, u)$, the blade assembly's motion is given by $u \exp (i \omega t)$, where $\omega$ is the complex frequency defined by $\lambda=\omega^{2}$ (and where $i^{2}=-1$ ). Writing $\omega=\omega_{R}+i \omega_{I}$, where $\omega_{R}$ is the frequency of oscillations and $\omega_{I}$ the damping in the aeroelastic mode considered, one can easily 
show that $\operatorname{Re} \lambda=\omega_{R}^{2}-\omega_{I}^{2}$ and $\operatorname{Im} \lambda=2 \omega_{I} \omega_{R}$. This means that for small damping the real and imaginary parts of $\lambda$ can be associated with the natural frequency and the damping in a mode, respectively. Note that instability, or flutter, occurs when $\omega_{I}<0$ or, equivalently, when the imaginary part of the eigenvalue $\lambda$ is negative. The flutter boundary is thus defined by $\omega_{I}=0$, or $\operatorname{Im} \lambda=0$.

Most of the numerical parameters used for generating the results reported in this paper are the same as those in the work of Kaza and Kielb. ${ }^{13}$ Only the differences are mentioned here, as follows. In most calculations the number of blades is $N=56$. The axial Mach number and the assumed vibration frequency used for aerodynamic computations are 0.641 and $\omega_{a}=238.08 \mathrm{~Hz}$, respectively. The rotational speed of the blade assembly is $3000 \mathrm{rpm}$. In the calculation of aerodynamic forces eight strips are used, and Table 1 gives the resulting spanwise Mach number distribution. Note that the tip Mach number is 1.103; thus, both subsonic and supersonic aerodynamic theories need to be used. The tip solidity is 2.382 and the tip mass ratio is 19.4569 . Only one component blade mode, the first torsion mode, is considered. This results in diagonal mass and stiffness matrices for the blade assembly and in a circulant aerodynamic matrix A. Furthermore, if we normalize the modes consistently so that all modal masses are equal, the mass matrix is proportional to the identity matrix, since in this study mistuning is introduced only in stiffness. For a tuned system the stiffness matrix is also proportional to the identity matrix.

In the rest of this paper we examine how the aeroelastic modes of the assembly obtained by solving Eq. (1) are affected by the introduction of small random blade mistuning. To achieve this we must first understand the dynamics of the perfectly tuned assembly. This is discussed in the next section.

\section{Cyclic Symmetry and Tuned Aeroelastic Eigensolution}

A tuned bladed-disk assembly features perfect cyclic symmetry in the sense that all blades are identical and the first blade (i.e., the reference blade) is adjacent to the $N$ th blade (i.e., the last blade). In the tuned case all matrices in Eq. (1) are block-circulant and the aeroelastic eigensolution has remarkable features. ${ }^{1,2}$ For a single-component mode per blade model the matrices become simply circulant. Important properties of circulant matrices are given in Appendix A. In this section we discuss the characteristics of the corresponding tuned aeroelastic modes.

\section{A. Aeroelastic Eigensolution of Tuned Assemblies}

The properties of circulant matrices (see Appendix A) determine the aeroelastic modes of tuned assemblies. Since $\mathbf{M}, \mathbf{K}$, and $\mathbf{A}$ in Eq. (1) are circulant, they share the same set of eigenvectors and thus the aeroelastic mode shapes of the tuned assembly are the $e_{j}, j=1, \ldots, N$, given in Eq. (A2). (This is true whether or not aerodynamic effects and/or structural coupling are included in the model.) This means that for a motion in the $j$ th mode all blades in the assembly vibrate with

Table 1 Spanwise Mach number distribution

\begin{tabular}{lc}
\hline \hline Strip no. & Mach no. \\
\hline 1 & 0.7163 \\
2 & 0.7393 \\
3 & 0.7830 \\
4 & 0.8466 \\
5 & 0.9230 \\
6 & 0.9998 \\
7 & 1.064 \\
8 & 1.103 \\
\hline
\end{tabular}

Strips are numbered from blade root to blade tip equal amplitudes and with a constant phase difference between adjacent blades. We rewrite this mode shape as

$$
\begin{gathered}
\boldsymbol{e}_{j}=\frac{1}{\sqrt{N}}\left[1, e^{i \sigma_{j}}, \ldots, e^{\left.i(N-1) \sigma_{j}\right]}\right]^{T} \\
\sigma_{j}=\frac{2 \pi(j-1)}{N}(\text { modulo } 2 \pi), \quad j=1, \ldots, N
\end{gathered}
$$

The phase angle $\sigma_{j}$ between adjacent blades is equal for all adjacent blade pairs. Consequently, the normal modes of the tuned assembly are referred to as constant interblade phase angle modes.

A motion in the $j$ th mode is that of a wave traveling through the assembly with a phase change $\sigma_{j}$ at each blade. It is shown in Appendix A that $\boldsymbol{e}_{N-j+2}=\overline{\boldsymbol{e}}_{j}$ (where an overbar denotes a complex conjugate); hence, a motion in the $(N-j+2)$ th mode is characterized by an interblade phase angle $\sigma_{N-j+2}=-\sigma_{j}$ (modulo $2 \pi$ ), corresponding to a wave traveling in a direction opposite to that of the $j$ th mode, with the same phase change at each blade in absolute value. Here we adopt the convention that a forward-traveling wave propagates in the direction of increasing blade number. Note that with this convention the positive rotation of the rotor is in the direction of backward-traveling waves, i.e., in the direction of decreasing blade number.

Hence we have the following description of the mode shapes:

1) The first mode shape, $\boldsymbol{e}_{1}$, corresponds to a zero interblade phase angle; all blades vibrate in phase with the same amplitude.

2) For even $N$, the $(N / 2+1)$ th mode has an interblade phase angle equal to $\pi$; adjacent blades vibrate out of phase with equal amplitudes.

3) Motions in the modes $\boldsymbol{e}_{j}$ corresponding to interblade phase angles $\left.\sigma_{j} \in\right] 0, \pi[$ are waves that travel backward through the assembly.

4) Motions in the modes $e_{j}$ such that $\left.\sigma_{j} \in\right] \pi, 2 \pi[$ are forward-traveling waves related to their backward-traveling counterparts by $\overline{\boldsymbol{e}}_{N-j+2}=\boldsymbol{e}_{j}$. A pair of forward- and backward-traveling waves have the same number of (traveling) nodal diameters.

The aerodynamic matrix $\mathbf{A}$ is complex; thus, from Eq. (A3), its eigenvalues are complex. This means that, if aerodynamic effects are included in the model, the aeroelastic eigenvalues of the system (1) are complex (recall that $\mathbf{M}$ and $\mathbf{K}$ are real). In addition, each pair of backward- and forward-traveling waves, $\boldsymbol{e}_{j}$ and $\overline{\boldsymbol{e}}_{j}$, is associated with two distinct eigenvalues, because the unsteady aerodynamic forces depend on the direction of rotation of the rotor. For example, since the backward traveling direction coincides with that of the rotor rotation, the aerodynamic interaction forces between two blades are different for a forward wave motion and a backward wave motion.

If aerodynamic effects are not included in the model, then $\mathbf{A}=\mathbf{0}$ and the eigenvalue problem (1) is real symmetric. When there is only structural coupling in a cyclic system, the eigenvalues are real and most eigenvalues have multiplicity 2 , such that $\lambda_{j}=\lambda_{N-j+2}$, except for the zero-interblade phase angle mode $(j=1)$ and, for $N$ even, for the $\pi$-interblade phase angle mode $(j=N / 2+1)$. The structural-only model has double eigenvalues because the direction of rotation of the assembly has no effect on its dynamics; thus, backward- and forwardtraveling waves cannot be distinguished. For each double eigenvalue these two traveling waves combine into standing waves with fixed nodal diameters. Furthermore, since the system studied herein features structurally uncoupled blades, removing the aerodynamic coupling yields $N$-fold degenerate eigenvalues for the tuned system.

The degeneracy that occurs in the cyclic structural system is removed by aerodynamic forces. It would also be removed by Coriolis forces if these were included in the formulation, since 
they depend on the direction of rotation (these forces are not considered here). Similarly, any infinitesimal amount of mistuning in one blade would also split the double eigenvalues of the structural system. The conclusion is that real physical rotors do not feature double eigenvalues.

Examples of motions in constant interblade phase modes are shown in Fig. 1 for an aeroelastic system. The waves travel along the assembly in the directions shown, except for the two standing-wave modes.

\section{B. Physical and Interblade Phase Angle Coordinates}

All matrices in Eq. (1) admit the $N$, independent, interblade phase angle modes as eigenvectors. Thus, we can diagonalize the tuned aeroelastic eigenvalue problem by introducing the coordinate transformation defined with the modal matrix $\mathbf{E}$ given in Eq. (A4). Let

$$
\boldsymbol{u}=\sum_{j=1}^{N} \eta_{j} \boldsymbol{e}_{j}=\mathbf{E} \boldsymbol{\eta}
$$

where the elements of $\boldsymbol{u}$ are the physical coordinates and where we define $\eta=\left[\eta_{1}, \ldots, \eta_{N}\right]^{T}$ as the vector of (assembly) modal coordinates, or interblade phase angle coordinates; $\eta_{j}$ represents the contribution of the mode with interblade phase angle $\sigma_{j}$ to the total motion of the assembly. The matrix $\mathbf{E}$ defines the transformation from interblade phase angle to physical coordinates.

First we apply this modal transformation to diagonalize the aerodynamic matrix. We have

$$
\tilde{\mathbf{A}}=\operatorname{diag}\left(\tilde{A}_{1}, \ldots, \tilde{A}_{j}, \ldots, \tilde{A}_{N}\right)=\mathbf{E}^{*} \mathbf{A E}
$$

because $\mathbf{E}$ is unitary, and where an asterisk denotes a conjugate transpose. The diagonal matrix $\widetilde{\mathbf{A}}$ is the matrix of eigenvalues of $\mathbf{A}$, made of the modal aerodynamic coefficients: $\tilde{A}_{j}$

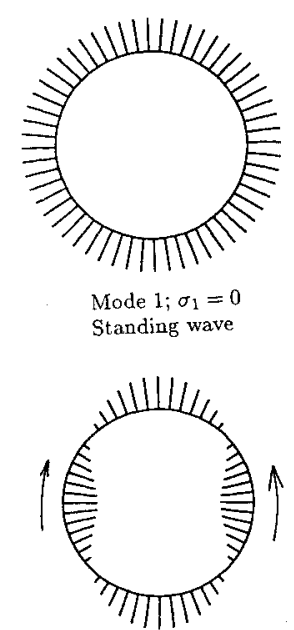

Modes 3 and $55 ; \sigma_{3}=-\sigma_{55}=\frac{4 \pi}{56}$ Traveling waves

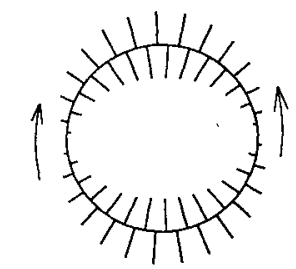

Modes 28 and $30 ; \sigma_{28}=-\sigma_{30}=\frac{2 \pi \times 27}{56}$ Traveling waves

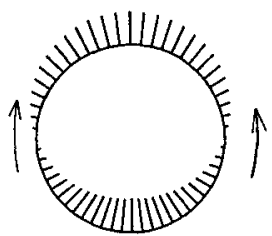

Modes 2 and $56 ; \sigma_{2}=-\sigma_{56}=\frac{2 \pi}{56}$ Traveling waves

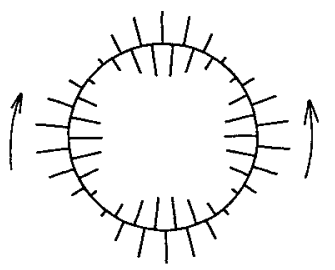

Modes 27 and $31 ; \sigma_{27}=-\sigma_{31}=\frac{2 \pi \times 26}{56}$ Traveling waves

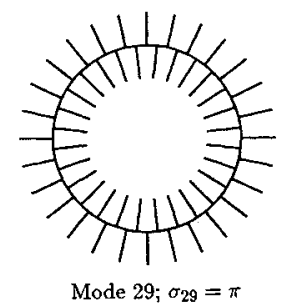

Standing wave
Fig. 1 Typical constant interblade phase angle modes of a tuned assembiy of 56 blades. The deflection pattern in the physical coordinates $u$ is shown at a given instant of time. Note the standing or traveling character of the mode shapes. Arrows indicate the opposite directions of travel of the waves. is the aerodynamic coefficient (e.g., a moment) for a cascade of blades oscillating in the $j$ th interblade phase angle mode. These modal aerodynamic coefficients are distinct as they depend on the interblade phase angle. In fact, it is typically $\tilde{\mathbf{A}}$, not $\mathbf{A}$, that is calculated by unsteady aerodynamic codes. This requires only $N$ independent calculations, one for each interblade phase angle. The generalized (modal) aerodynamic force for a motion of amplitude $\eta_{j}$ in the $j$ th interblade phase angle mode is $\tilde{A}_{j} \eta_{j}$, and the physical load on the blades is $\tilde{A}_{j} \eta_{j} \boldsymbol{e}_{j}$. For a general motion the physical load is a linear combination of the individual modal loads, given by $\mathbf{Q}_{A}=\mathbf{E} \tilde{\mathbf{A}} \eta$. From $\mathbf{Q}_{A}=\mathbf{A} u$ we retrieve Eq. (4) as $\mathbf{A}=\mathbf{E} \tilde{\mathbf{A}} \mathbf{E}^{*}$, where $\mathbf{A}$ contains the aerodynamic influence coefficients in the physical coordinates.

Next, the transformation from interblade phase angle to physical coordinates [Eq. (3)] can be applied to the eigenvalue problem [Eq. (1)], yielding

$$
\left\{-\lambda \mathbf{E}^{*} \mathbf{M E}+\mathbf{E}^{*} \mathbf{K E}-\tilde{\mathbf{A}}\right\} \boldsymbol{\eta}=\mathbf{0}
$$

where, for the tuned assembly considered here, $\mathbf{E}^{*} \mathbf{M E}=$ $\operatorname{diag}\left(M_{j}\right)$ and $\mathbf{E}^{*} \mathbf{K E}=\operatorname{diag}\left(K_{j}\right)$, where $M_{j}$ and $K_{j}$ are the generalized mass and stiffness for the $j$ th interblade phase angle mode, respectively. This gives the eigenvalues of the tuned assembly as

$$
\lambda_{j}=\frac{K_{j}-\tilde{A}_{j}}{M_{j}}, \quad j=1, \ldots, N
$$

Equation (6) can also be obtained by looking for the values of $\lambda$ such that the circulant matrix $(-\lambda \mathbf{M}+\mathbf{K}-\mathbf{A})$ has zero eigenvalues, by applying the general expression for the eigenvalues of a circulant matrix [Eq. (A3)].

Note that the imaginary part of $\lambda_{j}$, and thus the stability of the tuned assembly, is not affected by a change in the blade stiffness. Also note that the difference between any two eigenvalues is proportional to that between the corresponding modal aerodynamic coefficients.

\section{Nature of Aerodynamic Coupling and Structural Mistuning}

\section{A. Aerodynamic Matrix Characteristics}

Previous studies of structural models of blade assemblies (e.g., see Ref. 11) have shown that the key parameter that determines the sensitivity of their dynamics to mistuning is the interblade coupling. In our model the coupling between blades is provided solely by the aerodynamic terms. Thus, it appears reasonable to explore further the properties of the aerodynamic matrix in order to obtain useful insights into the effects of mistuning.

An important characteristic of $\mathbf{A}$ is that its elements are typically several orders of magnitude smaller than those of $\mathbf{K}$ and $\mathbf{M}$. This is because unsteady aerodynamic forces are very small compared to elastic and inertia forces, at least for the high-mass-ratio blade assemblies we are examining in this paper. It immediately follows that, although aerodynamic forces add stiffness and damping to the assembly, they do not change the natural frequencies of free oscillations much. The negative or positive aerodynamic damping that results is also small, although it may be sufficient to cause an instability. More important to our study, however, is the fact that, because the elements of $\mathbf{A}$ are small, the aerodynamic interblade coupling is weak for a typical assembly. Thus, according to earlier studies of mistuned structural systems and of mode localization, our model has the potential to feature dynamics that are highly sensitive to small mistuning. The small magnitude of the elements of $\mathbf{A}$ also suggests that perturbation schemes can be developed that treat the aerodynamic term as a perturbation. This will be useful when we attempt to characterize the localized modes.

The aerodynamic matrix is made up of influence coefficients: the element $A_{i j}$ is the generalized force on blade $i$ 
caused by a unit generalized displacement of blade $j$. Clearly, the aerodynamic interactions between two blades decrease as the distance between these two blades increases, and we can expect the coupling between adjacent blades to be the most significant. This feature is illustrated in Fig. 2, which displays the magnitude of the elements of one column of the matrix $\mathbf{A}$. We note the clear dominance of the terms closest to the diagonal and thus that of nearest-neighbor interblade coupling. The aerodynamic coupling with a next-to-neighboring blade is about one order of magnitude less than that with an adjacent blade.

This near-diagonal dominance of the aerodynamic matrix is yet another similarity with structural coupling, which is typically also strongest between adjacent blades. It suggests that the aerodynamic matrix in the physical coordinates can be reasonably approximated by a tridiagonal circulant or a pentadiagonal circulant matrix. (These matrices are not strictly trior pentadiagonal since they have elements near the upper-right and lower-left corners because of cyclicity.) Table 2 compares the least stable eigenvalue (the one with the smallest imaginary part) of the full matrix $\mathbf{A}$ with that of the tri- and pentadiagonal approximations. The comparison suggests that accounting for adjacent and next-to-adjacent blade coupling is sufficient to capture the assembly's dynamics accurately. (We have confirmed this conclusion by observing that the locus of the eigenvalues changes very little when we use the pentadiagonal approximation instead of the full matrix $\mathbf{A}$.) Such approximations of the aerodynamic matrix will be useful when we seek to characterize the localized aeroelastic modes in Sec. V.

\section{B. Structural Mistuning}

In this study we examine the effects of frequency mistuning only. We assume that the individual blade frequencies are random and uniformly distributed about the frequency of the nominal blade with a small standard deviation. We achieve this mistuning by altering the torsional stiffness of the blades. We only consider small random mistuning of standard deviation $<10 \%$, resulting from unavoidable manufacturing and material tolerances and wear.

For a mistuned assembly the stiffness matrix $\mathbf{K}$ is no longer circulant, but with our assumption of frequency mistuning, the mass and aerodynamic matrices do remain circulant. We show in the next section that this small deviation of $\mathbf{K}$ from perfect cyclicity results in the drastic alteration of the aeroelastic eigensolution, e.g., in the localization of the mode shapes. This high sensitivity originates from the weak interblade coupling terms in $\mathbf{A}$.

\section{Perturbation Analysis of Mistuned Assemblies}

In this section we attempt to predict and characterize mistuning effects on the aeroelastic eigensolution through the use of perturbation schemes. We first apply a standard, or classical, perturbation method that predicts the high sensitivity to mistuning. Then we develop a perturbation scheme that is able

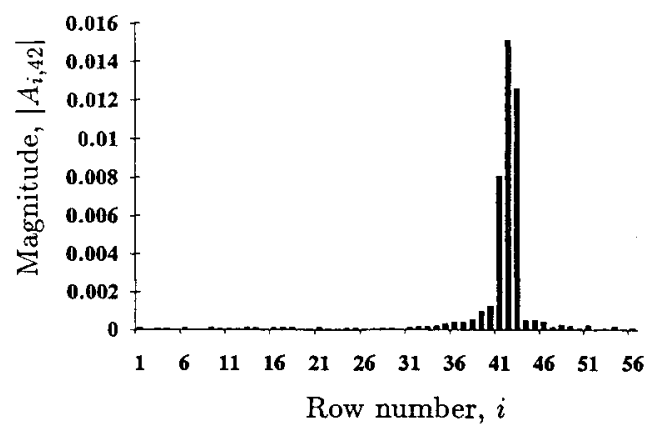

Fig. 2 Magnitude of the elements of the 42 nd column of the aerodynamic matrix in the physical coordinates $A$ for the parameters in Sec. II. Note the dominance of the near-diagonal elements.
Table 2 Real and imaginary parts of the least stable eigenvalue of a tuned assembly, obtained with the full aerodynamic matrix $A$ and with the tridiagonal-circulant and pentadiagonal-circulant truncations of $A$

\begin{tabular}{lcc}
\hline \hline Matrix & $\operatorname{Re} \lambda_{U}$ & $\operatorname{Im} \lambda_{U}$ \\
\hline Full & 1.2025 & 0.0077 \\
Pentadiagonal & 1.2016 & 0.0066 \\
Tridiagonal & 1.2041 & 0.0042 \\
\hline \hline
\end{tabular}

to handle large mistuning effects and thus characterize the mistuned eigensolutions (e.g., the localized modes).

\section{A. Prediction of High Sensitivity to Mistuning}

Even though classical perturbation methods fail to describe the dynamics of a mistuned assembly when it is qualitatively different from that of the corresponding tuned system, they predict high sensitivity and provide useful insight into the onset of mode localization. (We refer the reader who is unfamiliar with perturbation theory for the eigenvalue problem to Ref. 16.) In the classical approach the unperturbed system is the tuned assembly and the perturbation is the frequency mistuning. The unperturbed eigenvalue problem is

$$
\left\{-\lambda_{o} \mathbf{M}+\mathbf{K}_{o}-\mathbf{A}\left(\omega_{a}\right)\right\} \boldsymbol{u}_{o}=\mathbf{0}
$$

where, for no structural coupling and one component mode per blade, $\mathbf{M} \equiv M \mathbf{I}$ and $\mathbf{K}_{o} \equiv K_{o} \mathbf{I}$. From Eq. (6), the unperturbed eigenvalues are $\lambda_{\mathrm{o} j}=\left(K_{o}-\tilde{A}_{j}\right) / M$, and the unperturbed aeroelastic mode shapes are $u_{o j}=e_{j}(j=1, \ldots, N)$. Note that the eigenvalues are clustered in a narrow band because the modal aerodynamic coefficients are small. Also, the imaginary parts of the eigenvalues (representing the damping) are small because the $\widetilde{A}_{j}$ are small.

For the mistuned system the stiffness matrix becomes

$$
\mathbf{K}=\mathbf{K}_{o}+\delta \mathbf{K}, \quad \delta \mathbf{K}=\operatorname{diag}\left(\delta K_{1}, \ldots, \delta K_{i}, \ldots, \delta K_{N}\right)
$$

where $\delta K_{i}$ is the deviation of the $i$ th blade stiffness from the nominal value $K_{o}$, such that $\delta K_{i} / K_{o} \ll 1$. We assume the $\delta K_{i}$ are independent and identical random variables of mean zero and standard deviation $\sigma$. In this paper we consider only one (arbitrary) pattern of random mistuning, such that

$$
\left\{\begin{array}{l}
\frac{1}{N} \sum_{i=1}^{N} \delta K_{i} \simeq 0 \\
\frac{1}{N-1} \sum_{i=1}^{N} \delta K_{i}^{2}=\epsilon^{2} \simeq \sigma^{2}
\end{array}\right.
$$

i.e., the estimates of the mean and the standard deviation obtained from one realization of mistuning are close to the mean and standard deviation of the mistuning random variable, respectively. With this notation the mistuning is order $\epsilon$, and with the assumption of zero mean the average stiffness of the mistuned assembly's blades is the nominal blade stiffness.

The perturbed (mistuned) eigenvalue problem is given by Eq. (1). We consider a second-order perturbation expansion of the eigensolution as

$$
\left\{\begin{array}{l}
\lambda_{j}=\lambda_{o j}+\delta \lambda_{j}+\delta^{2} \lambda_{j} \\
u_{j}=u_{o j}+\delta u_{j}+\delta^{2} u_{j}
\end{array} \quad j=1, \ldots, N\right.
$$

where $\delta \lambda_{j}$ and $\delta \boldsymbol{u}_{j}$ (respectively $\delta^{2} \lambda_{j}$ and $\delta^{2} \boldsymbol{u}_{j}$ ) are first-order (respectively second-order) terms in $\epsilon$. The general perturbation formulas are given in Appendix B.

1. First-Order Eigenvalue Perturbation

We can show, using Eq. (B5), that

$$
\begin{aligned}
\delta \lambda_{j} & =\frac{1}{M}\left(e_{j}^{*} \delta \mathbf{K} \boldsymbol{e}_{j}\right) \\
& =\frac{1}{M} \frac{1}{N} \operatorname{tr}(\delta \mathbf{K}) \approx 0, \quad j=1, \ldots, N
\end{aligned}
$$


where tr denotes the trace of a matrix, the sum of its diagonal elements. We make two observations. 1) The $\delta \lambda_{j}$ is real. This means that there is no first-order effect of mistuning on the flutter boundary, because stability is only affected by the imaginary part of the eigenvalues. 2) The first-order perturbation $\delta \lambda_{j}$ is independent of $j$. Hence, all the eigenvalues are shifted identically as a result of mistuning, by an amount equal to the average of the deviations of the frequencies squared from the nominal value. For small mistuning this eigenvalue shift is always small. Moreover, in this paper the mistuning pattern has a (nearly) zero mean; thus, the first-order effect on the eigenvalues is (nearly) equal to zero. We conclude that $\delta \lambda_{j}$ is at most a term of order $\epsilon$ that cannot reveal high sensitivity to mistuning.

\section{High Mode Shape Sensitivity}

The first-order effect of mistuning on the aeroelastic mode shape is, from Eq. (B7),

$$
\begin{aligned}
\delta \boldsymbol{u}_{j} & =\sum_{\substack{k=1 \\
k \neq j}}^{N} \frac{1}{\lambda_{o j}-\lambda_{o k}} \frac{1}{M}\left(\boldsymbol{e}_{k}^{*} \delta \mathbf{K} \boldsymbol{e}_{j}\right) \boldsymbol{e}_{k} \\
& =\sum_{\substack{k=1 \\
k \neq j}}^{N} \frac{\boldsymbol{e}_{k}^{*} \delta \mathbf{K} \boldsymbol{e}_{j}}{\tilde{A}_{k}-\tilde{A}_{j}} \boldsymbol{e}_{k}
\end{aligned}
$$

where we use Eq. (6). Equation (12) tells us that the magnitude of $\delta u_{j}$ is determined by the ratios $\left(\boldsymbol{e}_{k}^{*} \delta \mathbf{K} \boldsymbol{e}_{j}\right) /\left(\tilde{A}_{k}-\tilde{A}_{j}\right)$. If all eigenvalues are well separated, then $\delta \boldsymbol{u}_{j}$ is effectively first order. However, if $\left|\tilde{A}_{k}-\tilde{A}_{j}\right|$ is order $\epsilon$ or smaller, then $\delta u_{j}$ is not order $\epsilon$ any longer, but of the order of 1 or larger. The assumptions for the use of asymptotic expansions in perturbation theory are then violated, and the perturbation analysis fails, thereby indicating that the mode shapes undergo dramatic changes.

We have seen in Sec. IV that the aerodynamic coupling is typically weak; thus, the denominators in Eq. (12), $\bar{A}_{k}-\bar{A}_{j}$, are first-order or smaller terms. (This is qualitatively similar to the structural case where the distance between eigenvalues is also small for small interblade coupling.) This means that for first-order mistuning the ratio of disorder to interblade coupling, and thus $\delta u_{j}$ [see Eq. (12)], is finite or large, and that the aeroelastic mode shapes are highly sensitive to mistuning. We show in Sec. V.C that this failure of the perturbation analysis indicates the occurrence of mode localization.

It is interesting to note that the quantity $\boldsymbol{e}_{k}^{*} \delta \mathbf{K} \boldsymbol{e}_{j}(k \neq j)$ is the coupling of the unperturbed eigenvectors through the mistuning matrix and provides a good representation of disorder in the assembly. For example, if the stiffness of all blades were changed by the same amount, then $\delta \mathbf{K}$ would be proportional to the identity matrix and there would be no disorder since $\boldsymbol{e}_{k}^{*} \mathbf{I} \boldsymbol{e}_{j}=0$.

\section{B. Eigenvalue Loci Veering Phenomena}

While the first-order mode shape perturbations provide insight into the sensitivity to mistuning, interesting and useful behavior can also be observed by considering the second-order eigenvalue perturbations. From Eq. (B6),

$$
\delta^{2} \lambda_{j}=\frac{1}{M} \sum_{\substack{k=1 \\ k \neq j}}^{N} \frac{\left|e_{k}^{*} \delta \mathbf{K} \boldsymbol{e}_{j}\right|^{2}}{\tilde{A}_{k}-\tilde{A}_{j}}, \quad j=1, \ldots, N
$$

where vertical bars denote the modulus of a complex number. The same mechanisms as those described earlier for the mode shapes lead to high sensitivity. Namely, $\delta^{2} \lambda_{j}$ is not second order, but first order or larger, when the coupling of the eigenvectors through the mistuning $\boldsymbol{e}_{k}^{*} \delta \mathbf{K} \boldsymbol{e}_{j}$ is of the same order or larger than the aerodynamic coupling, measured by $\left|\tilde{A}_{k}-\tilde{A}_{j}\right|$.Then perturbation theory fails. Note that these large mistuning effects are predicted by the second-order eigenvalue perturbation but are completely overlooked by the first-order perturbation [Eq. (11)].
From Eq. (13) we conjecture that the sensitivity to mistuning increases with the number of blades. This is caused by two mechanisms: 1) As $N$ increases the number of interblade phase angles increases and the difference between two adjacent ones, $2 \pi / N$, decreases. In turn, we can reasonably expect the difference between the two corresponding modal aerodynamic coefficients to decrease and thus the terms in the summation (13) to increase. 2) As $N$ increases the number of terms in Eq. (13) increases and thus the second-order eigenvalue perturbation increases. This is readily seen for the eigenvalues such that all terms in the summation are positive or negative (e.g., for the real part of the lowest- and highest-frequency eigenvalues and for the imaginary part of the least and most stable eigenvalues). This larger effect of mistuning with increasing number of blades is illustrated in Sec. VI.

Another remark is that $\delta^{2} \lambda_{j}$ is complex and thus both frequencies and damping values are affected by mistuning to the second order, unlike the first order. Below we examine in detail the real and the imaginary parts of the mistuned eigenvalues. Recall that they correspond to the frequency and damping in an aeroelastic mode, respectively.

\section{Veering A way of the Frequency Loci}

From Eq. (13), the real part of the second-order perturbation is

$$
\begin{array}{r}
\operatorname{Re} \delta^{2} \lambda_{j}=\frac{1}{M} \sum_{\substack{k=1 \\
k \neq j}}^{N} \frac{\operatorname{Re} \tilde{A}_{k}-\operatorname{Re} \tilde{A}_{j}}{\left|\tilde{A}_{k}-\tilde{A}_{j}\right|^{2}}\left|e_{k}^{*} \delta \mathbf{K} e_{j}\right|^{2} \\
j=1, \ldots, N
\end{array}
$$

Consider the locus of the real part of the $j$ th eigenvalue $\operatorname{Re} \lambda_{j}$ vs the mistuning strength as estimated by the standard deviation, $\epsilon$. From Eq. (14), $\operatorname{Re} \delta^{2} \lambda_{j}$ is proportional to $\epsilon^{2}$, the coefficient of proportionality being equal to one-half the curvature of the locus of $\operatorname{Re} \lambda_{j}$ vs $\epsilon$. We observe from Eq. (14) that this curvature is inversely proportional to the interblade coupling and is thus large for weak aerodynamic coupling.

Now consider the tuned eigenvalues with the smallest and largest real parts, corresponding to the modes with the lowest and highest natural frequencies, and denote them $\lambda_{L}$ and $\lambda_{H}$. From Eq. (6), these two eigenvalues are associated with the modal aerodynamic coefficients with the largest and smallest real parts, respectively. (Note that the interblade phase angles corresponding to the eigenvalues with extreme real parts depend on the system studied and its parameters.) For the low-

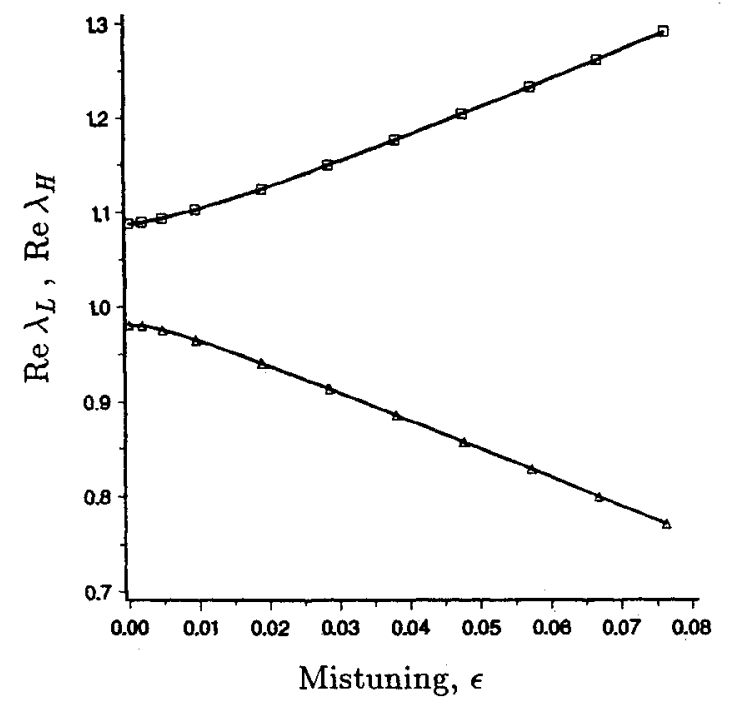

Fig. 3 Loci of the real parts (frequency) of the eigenvalues corresponding to the highest- and lowest-frequency modes vs mistuning strength. Observe the abrupt veering away of the loci for small mistuning. 
est-frequency eigenvalue all terms in the summation, Eq. (14), are negative; hence, $\operatorname{Re} \delta^{2} \lambda_{L}<0$ and the locus of the real part of the lowest frequency eigenvalue has a large negative curvature at the tuned state. Similarly, for the eigenvalue with the largest real part, all terms in the summation are positive. Thus, $\operatorname{Re} \delta^{2} \lambda_{H}>0$ and the highest frequency locus has a large positive curvature. This means that the loci of the extreme real parts, $\operatorname{Re} \lambda_{L}$ and $\operatorname{Re} \lambda_{H}$, have large and opposite curvatures: the loci abruptly veer away from each other at the tuned state $\epsilon=0$. This eigenvalue loci veering is illustrated in Fig. 3 for the system parameters of Sec. II. It indicates the high sensitivity to mistuning and is the same phenomenon that was observed previously for the lowest and highest frequencies of a structural assembly. ${ }^{11}$

The phenomenon of veering away of the loci suggests that second-order eigenvalue perturbations can be used effectively to indicate high sensitivity. It also tells us that the modes with extreme frequencies are more sensitive to mistuning than the other modes and thus will localize first, because the corresponding loci have larger curvatures.

\section{Veering Toward of the Damping Loci}

Now consider the effects of mistuning on the imaginary part of the eigenvalues. We have, from Eq. (13),

$$
\begin{array}{r}
\operatorname{Im} \delta^{2} \lambda_{j}=\frac{1}{M} \sum_{\substack{k=1 \\
k \neq j}}^{N} \frac{\operatorname{Im} \tilde{A}_{j}-\operatorname{Im} \tilde{A}_{k}}{\left|\tilde{A}_{k}-\tilde{A}_{j}\right|^{2}}\left|\boldsymbol{e}_{k}^{*} \delta \mathbf{K} \boldsymbol{e}_{j}\right|^{2} \\
j=1, \ldots, N
\end{array}
$$

Recall that $\operatorname{Im} \lambda_{j}$ determines the damping in the $j$ th mode, with flutter occurring if $\operatorname{Im} \lambda_{j} \leq 0$. The least stable mode of the tuned assembly corresponds to the eigenvalue with the smallest imaginary part $\lambda_{U}$, which in turn is associated with the interblade phase angle that yields the modal aerodynamic coefficient with the largest imaginary part [from Eq. (6)]. Thus, all terms in the summation, Eq. (15), are positive for the least stable eigenvalue and $\operatorname{Im} \delta^{2} \lambda_{U}>0$. It follows that mistuning increases the imaginary part of the least stable eigenvalue (because $\delta \lambda_{j}=0$ for all $j$ ); hence, it has a stabilizing effect. This beneficial effect of mistuning on the least stable root holds for any mistuning pattern, provided the average blade frequency is not altered by mistuning, i.e., $\Sigma_{i=1}^{N} \delta K_{i}=0$. This finding agrees with that of Bendiksen. ${ }^{7}$ We point out, however, that the stabilizing effect of mistuning does not necessarily hold for the other eigenvalues, because some of the terms $\left(\operatorname{Im} \tilde{A}_{j}-\operatorname{Im} \tilde{A}_{k}\right)$ in the summation (15) are negative. For example, the most stable root becomes less stable. It is conceivable, at least mathematically, that for some mistuning pattern an eigenvalue near the least stable one could become unstable (although we never encountered such a case numerically). Thus, care should be exerted when the stabilizing effect of mistuning on flutter is exploited.

Since aerodynamic forces are small, $\operatorname{Im} \delta^{2} \lambda_{U}$, although nominally a second-order term, is first order or larger, which means that mistuning has a first-order or larger effect on the flutter speed (again this agrees with the findings of Ref. 7). This makes the use of mistuning as a means of passive flutter control attractive. However, it should be pointed out that Eq. (15) is not a valid approximation of the least stable mistuned root in this high sensitivity case, because it is precisely the failure of the perturbation expansion that indicates the large mistuning effects. To obtain a correct approximation of the mistuned eigenvalues, we must use the modified perturbation scheme presented in Sec. V.C.

Next, we consider the loci of the imaginary parts of the eigenvalues $\operatorname{Im} \lambda_{j}$ vs the mistuning strength $\epsilon$. Expectedly, Im $\delta^{2} \lambda_{j}$ is proportional to the curvature of the $j$ th locus at the tuned state. The least and most stable tuned roots, $\lambda_{U}$ and $\lambda_{S}$, are those with the smallest and largest imaginary parts and correspond to the modal aerodynamic coefficients with the largest and smallest imaginary parts, respectively. From Eq.
(15) we deduce readily that $\operatorname{Im} \delta^{2} \lambda_{U}>0$ and $\operatorname{Im} \delta^{2} \lambda_{S}<0$. Hence, for weak interblade coupling the curvatures of the loci of Im $\lambda_{U}$ and Im $\lambda_{S}$ are large and opposite. However, contrary to the loci of the real parts, the locus of the root with the smallest imaginary part has positive curvature and vice versa. It follows that the loci of the two extreme imaginary parts veer toward each other with large curvature at the tuned state. This phenomenon is illustrated in Fig. 4, which displays the imaginary parts of the least and most stable roots vs mistuning for the parameters of Sec. II. We believe the phenomenon of the damping loci veering toward is characteristic of highly sensitive aeroelastic systems and has not been examined before.

\section{Analysis of Mode Localization by Modified Perturbation Methods}

Once high sensitivity has been predicted by the perturbation approach described earlier, the next step is to analyze the characteristics of the aeroelastic modes of the mistuned system. To do so, perturbation methods have to be modified to handle the dramatic changes resulting from small mistuning. Such an approach has been developed in Ref. 11 to analyze localization in structurally coupled assemblies.

The key idea behind the modified perturbation scheme is to recognize that high sensitivity is caused by the small interblade coupling; hence, this small parameter should be treated as a perturbation. However, if both mistuning and coupling are considered perturbations, the unperturbed system consists of uncoupled identical blades and thus is $N$-fold degenerate. To remove this degeneracy, we include mistuning in the unperturbed state and treat the interblade coupling as the perturbation. With this modified perturbation scheme the unperturbed system is purely structural, consisting of uncoupled mistuned blades in a vacuum. It thus has distinct natural frequencies (unless two mistuned blades happen to have the same frequency, but we shall not consider this unlikely case). The perturbation consists of the small unsteady aerodynamic forces. Each normal mode of the unperturbed system features uncoupled oscillations of a single mistuned blade, with all other blades remaining quiescent. When weak interblade coupling is introduced, the neighboring blades participate in the modal motion, but do so with small amplitudes because the small coupling is not sufficient to cause a resonance among the slightly different blades. Each mode of the mistuned assembly is a perturbation of the oscillations of a single blade, and thus is localized to that blade or to the small neighboring geometric region, depending on the magnitudes of coupling and mistuning. In the following we formalize this description of localization.

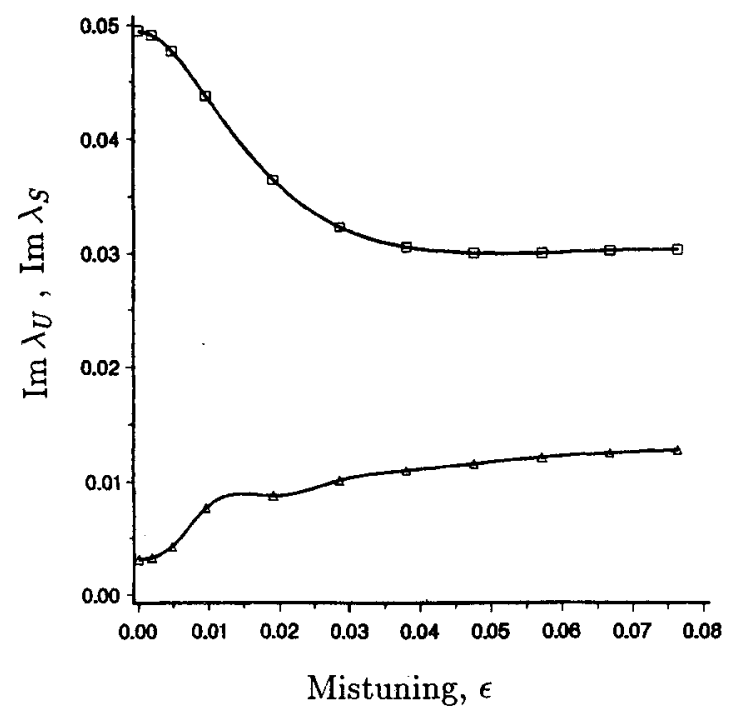

Fig. 4 Loci of the imaginary parts (damping) of the least and most stable eigenvalues vs mistuning strength. Observe the rapid veering of the loci as mistuning increases. 


\section{Effect of Aerodynamic Coupling on Eigenvalues}

With the modified perturbation scheme the unperturbed eigenvalue problem is

$$
\left\{-\lambda_{o}^{(m)} \mathbf{M}+\mathbf{K}_{o}+\delta \mathbf{K}\right\} u_{o}^{(m)}=\mathbf{0}
$$

where the superscript $m$ refers to the modified perturbation method. Since all matrices in Eq. (16) are diagonal, the modified unperturbed eigensolutions are simply

$$
\left\{\begin{array}{l}
\lambda_{o i}^{(m)}=\frac{K_{o}+\delta K_{i}}{M} \\
\boldsymbol{u}_{o i}^{(m)}=\mathbf{1}_{i}=[0, \ldots, 0, \underbrace{1}_{\text {position } i}, 0, \ldots, 0]^{T}
\end{array} i=1, \ldots, N\right.
$$

corresponding to purely localized oscillations of individual blades at their (rotating) mistuned frequencies. (Note that these eigenvalues are sorted according to blade number, not by increasing frequency.)

The matrix ( $-\mathbf{A}$ ) is the modified perturbation that provides the aerodynamic interblade coupling. From Eq. (B5) one can show that the first-order modified eigenvalue perturbation is

$$
\delta \lambda_{i}^{(m)}=-\frac{A_{i i}}{M}, \quad i=1, \ldots, N
$$

Since the aerodynamic matrix in the physical coordinates $\mathbf{A}$ is circulant, it has identical diagonal elements, and one can easily show that

$$
A_{i i}=\frac{1}{N} \operatorname{tr} \mathbf{A}=\frac{1}{N} \operatorname{tr} \tilde{\mathbf{A}}, \quad i=1, \ldots, N
$$

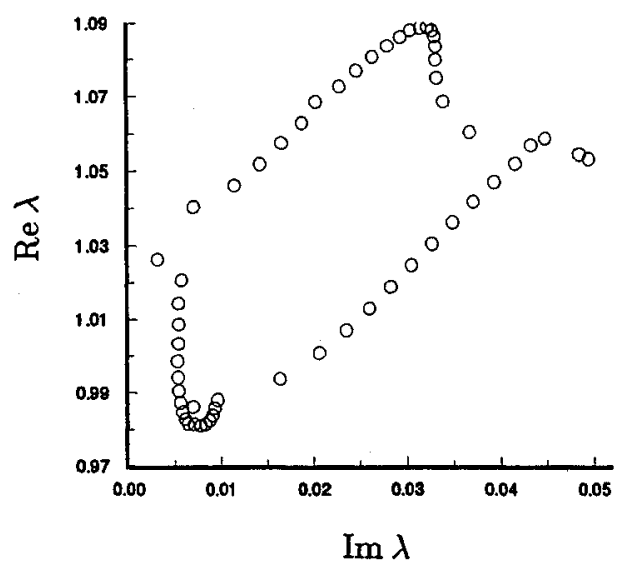

a) Tuned, $\epsilon=0$

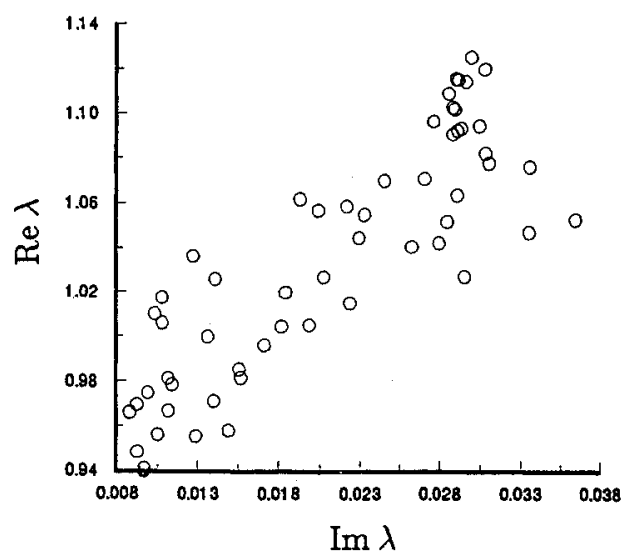

c) Mistuned, $\epsilon=1.90 \%$ since the trace is an invariant under similarity transformation. Equations (18) and (19) mean that to the first order the eigenvalues are displaced by the average of the modal aerodynamic coefficients, which provides both damping and additional stiffness to each mode. A similar finding was obtained by Wei and Pierre, ${ }^{11}$ who showed that for weak structural coupling the eigenvalues are shifted approximately by an amount equal to the coupling stiffness between the blades.

To the first order in the aerodynamic effects, the eigenvalues of the mistuned assembly are

$$
\lambda_{i} \simeq \frac{1}{M}\left[K_{o}+\delta K_{i}-\frac{1}{N} \operatorname{tr} \tilde{\mathbf{A}}\right], \quad i=1, \ldots, N
$$

This approximation holds for small values of the ratio of aerodynamic coupling to frequency mistuning, that is, for not-too-small mistuning. It is not valid in the range of very small mistuning values where curve veering phenomena and high sensitivity occur. In this region the classical perturbation method described in Secs. V.A and V.B approximates the dynamics well.

Now consider the loci of the real and imaginary parts of the eigenvalues vs mistuning strength $\epsilon$. To the first order we have $\operatorname{Re} \lambda_{i} \simeq\left(K_{o}+\delta K_{i}-\operatorname{Re}(\operatorname{tr} \tilde{\mathbf{A}}) / N\right) / M$. This tells us that, away from the veering region, the loci of the real parts tend to straight lines whose slopes are determined by the individual blade mistunings. This trend is indeed observed in Fig. 3 for not-too-small mistuning. For the imaginary parts we have $\operatorname{Im} \lambda_{j} \simeq-\operatorname{Im}(\operatorname{tr} \tilde{\mathbf{A}}) / N M$; thus, we expect the loci to approach a straight line with zero slope as mistuning increases. This is indeed observed in Fig. 4, although the imaginary parts of the eigenvalues tend to distinct values, whereas the first-order result [Eq. (20)] predicts identical asymptotes.

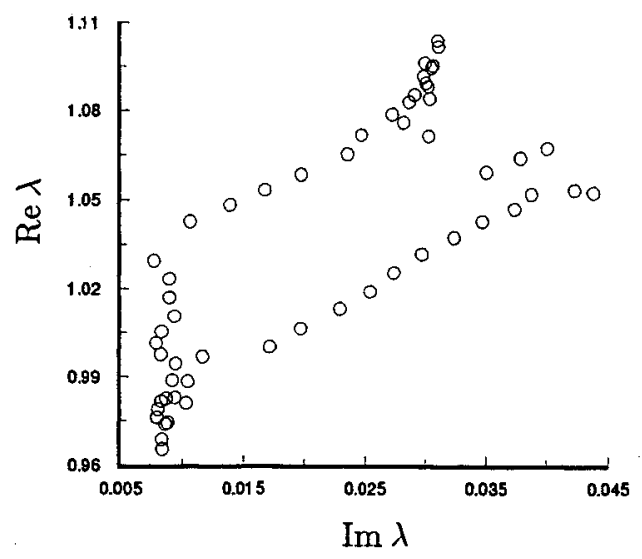

b) Mistuned, $\epsilon=\mathbf{0 . 9 5 \%}$

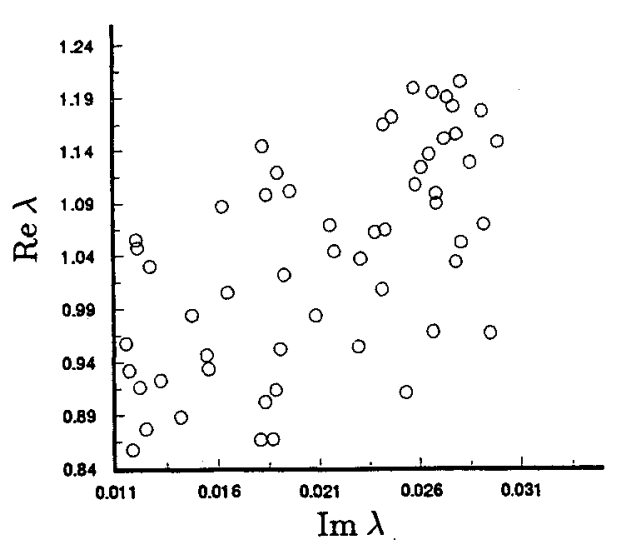

d) Mistuned, $\epsilon=4.76 \%$

Fig. 5 Root locus of the 56 aeroelastic eigenvalues in the complex plane (frequency vs damping) for various values of blade mistuning. The regular root locus of the tuned assembly becomes scattered in an apparently random fashion as mistuning increases. 

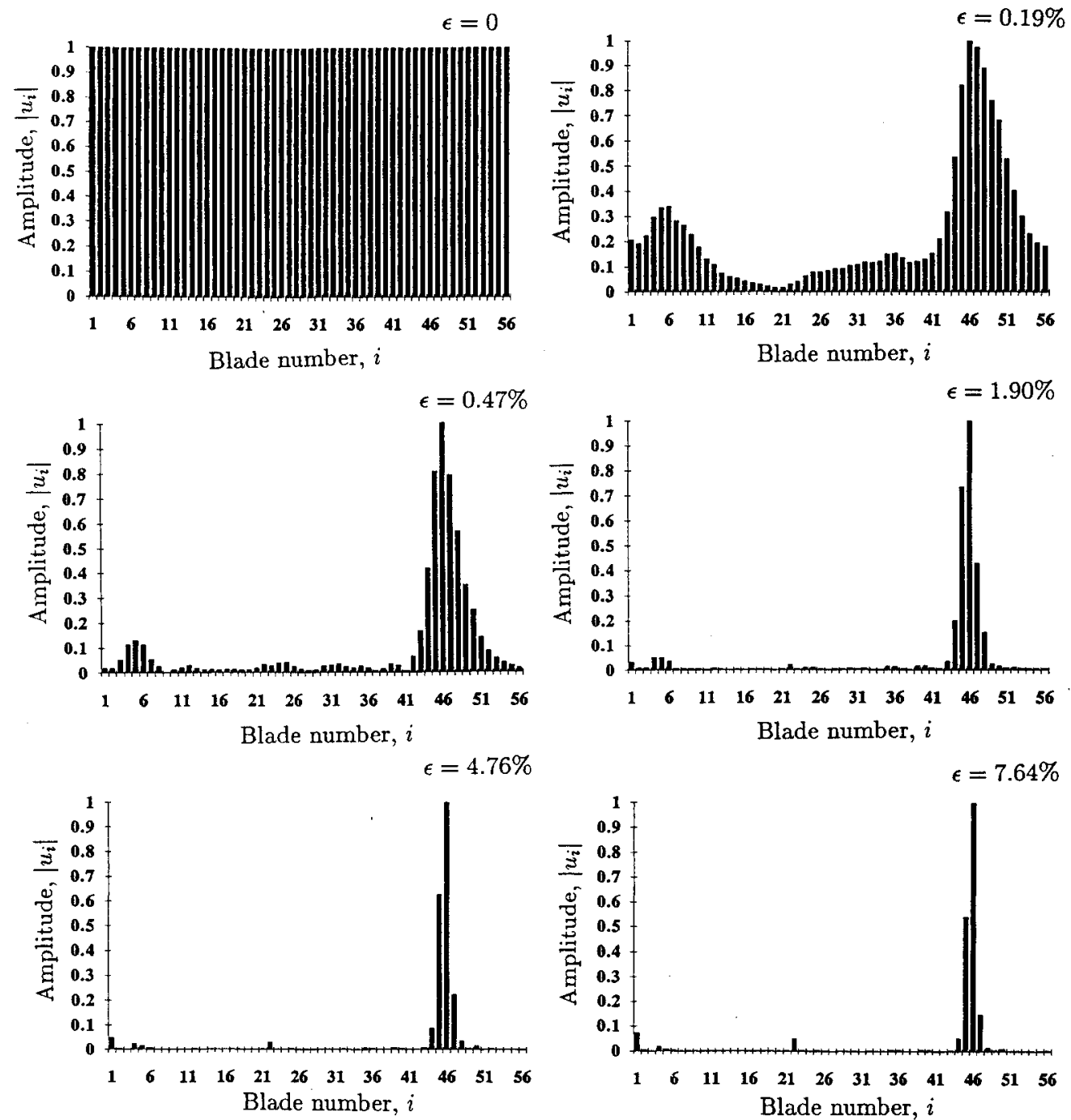

Fig. 6a Aeroelastic mode shape associated with the lowest-frequency eigenvalue $\lambda_{L}$ for various mistuning strengths and patterns of amplitudes. The extended mode of the tuned system becomes severely localized for small mistuning and loses its constant interblade phase angle.

We can improve our approximation by including second-order effects in the aerodynamic coupling. This yields

$$
\delta^{2} \lambda_{i}^{(m)}=\frac{1}{M} \sum_{\substack{k=1 \\ k \neq i}}^{N} \frac{A_{k i} A_{i k}}{\delta K_{i}-\delta K_{k}}, \quad i=1, \ldots, N
$$

Note that since $\mathbf{A}$ is circulant, there are only $N$ distinct elements $A_{i k}$. These perturbation results are compared with numerical solutions in Sec. VI.

\section{Localized Aeroelastic Mode Shapes}

Using Eq. (B7), we obtain the first-order modified perturbations of the eigenvectors as

$$
\delta \boldsymbol{u}_{i}^{(m)}=-\sum_{\substack{k=1 \\ k \neq i}}^{N} \frac{\mathbf{1}_{k}^{T} \mathbf{A} \mathbf{1}_{i}}{\delta K_{i}-\delta K_{k}} \mathbf{1}_{k}, \quad i=1, \ldots, N
$$

where $\mathbf{1}_{k}$ is defined in Eq. (17). Combining Eq. (22) with Eq. (17), we obtain the approximate mode shapes to the first order in the aerodynamic coupling:

$$
\begin{aligned}
u_{i}= & {[\frac{A_{1 i}}{\delta K_{1}-\delta K_{i}}, \ldots, \frac{A_{i-1, i}}{\delta K_{i-1}-\delta K_{i}} \underbrace{1}_{\text {position } i},} \\
& \left.\frac{A_{i+1, i}}{\delta K_{i+1}-\delta K_{i}}, \ldots, \frac{A_{N i}}{\delta K_{N}-\delta K_{i}}\right]^{T} \quad i=1, \ldots, N
\end{aligned}
$$

Several remarks are in order:

1) The modified perturbation method is valid for small aerodynamic coupling to mistuning ratio. This means that all but the $i$ th element of the $i$ th mode shape in Eq. (23) are much smaller than one. Hence, the $i$ th aeroelastic mode shape is localized about the $i$ th blade. The other blades participate in the modal motion, but with much smaller amplitudes. It is important to note that localization occurs for mistuning that is not too small, i.e., away from the veering region in the eigenvalue plots (Figs. 3 and 4 ).

2) We observe in Eq. (23) that the vibration amplitude of a blade in a localized mode is directly proportional to the amount of aerodynamic coupling between that blade and the large amplitude blade. Since we have seen in Sec. IV that the coupling between two blades decreases rapidly as the distance between them increases, it suggests that only the blades that are close to the large amplitude blade vibrate with an amplitude that is not negligible. In other words, there is a rapid spatial decay of the blade amplitudes away from the large amplitude blade, and for a strongly localized mode only the nearest neighboring blades participate in the motion.

3) In a localized mode the vibration amplitude of a blade is inversely proportional to the difference between its stiffness and that of the large amplitude blade. Hence, two blades that are far apart but whose frequencies are sufficiently close may experience local resonances, even though the blades between those two vibrate with very small amplitudes. This means that depending on the mistuning pattern for the assembly a mode may be localized about more than one blade. 

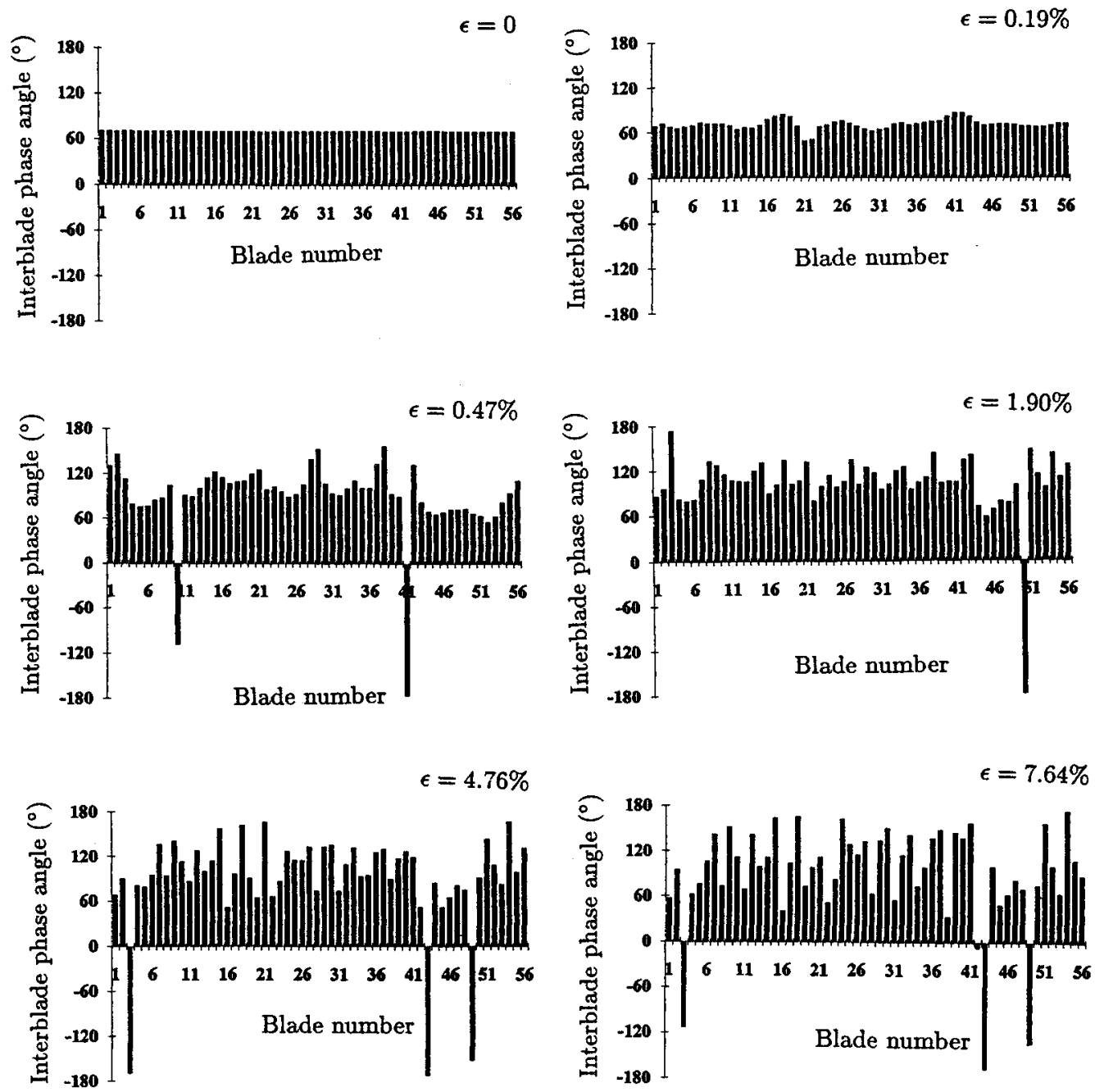

Fig. 6b Aeroelastic mode shape associated with the lowest-frequency eigenvalue $\lambda_{L}$ for various mistuning strengths and patterns of interblade phase angles. The extended mode of the tuned system becomes severely localized for small mistuning and loses its constant interblade phase angle.

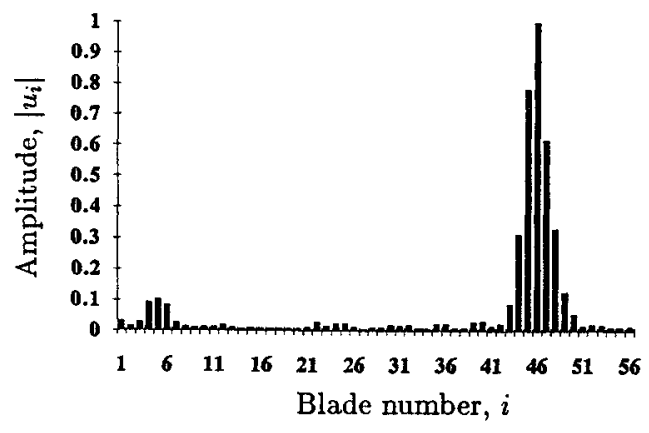

a) Lowest frequency mode; $\lambda_{L}=\lambda_{1}$

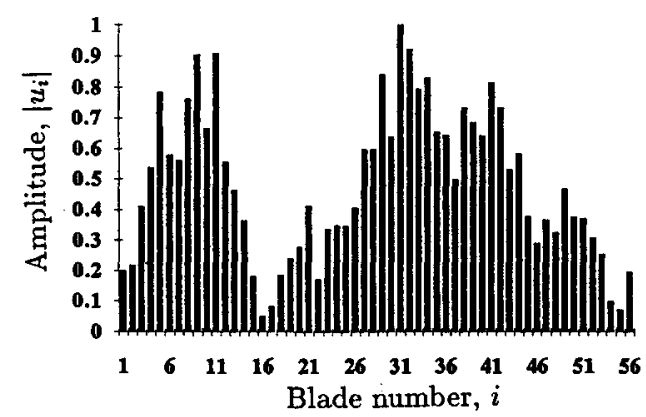

c) Most stable mode; $\lambda_{S}=\lambda_{34}$

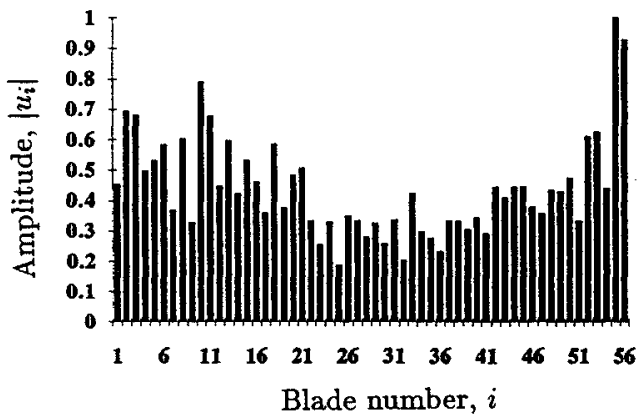

b) Least stable mode; $\lambda_{U}=\lambda_{26}$

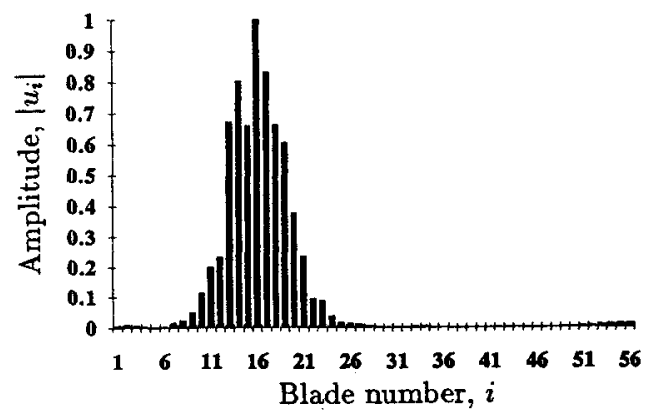

d) Highest frequency mode; $\lambda_{H}=\lambda_{56}$

Fig. 7 Amplitude patterns of aeroelastic mode shapes of an assembly with $\epsilon=0.95 \%$ mistuning. The lowest- and highest-frequency modes and the least and most stable modes are shown. Observe that the modes near the edges of the frequency cluster (modes 1 and 56 ) are more localized than those near the middle of the frequency band (modes 26 and 34). 


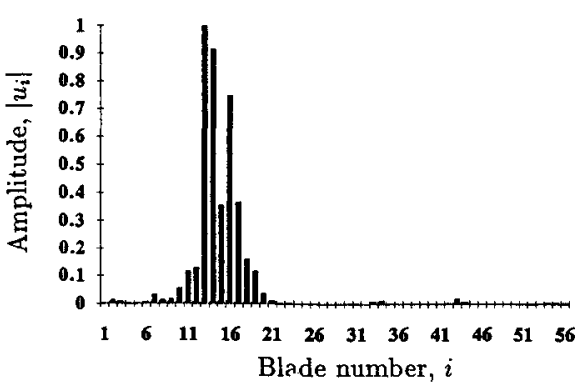

a) 56-blade assembly

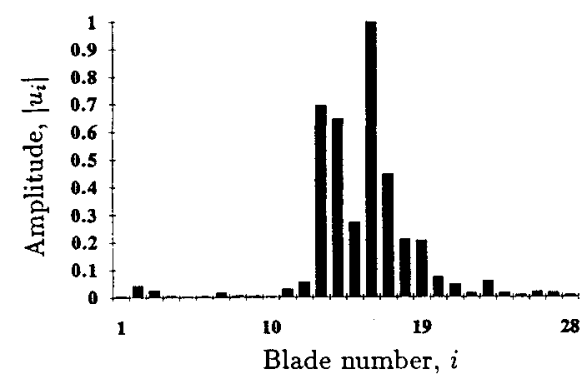

b) 28-blade assembly

Fig. 8 Amplitude pattern of the highest-frequency mode $\lambda_{H}$ for mistuned assemblies with 28 and 56 blades. The mistuning strength is $\epsilon=\mathbf{2 . 8 5} \%$. Note the more severe localization for $N=\mathbf{5 6}$.

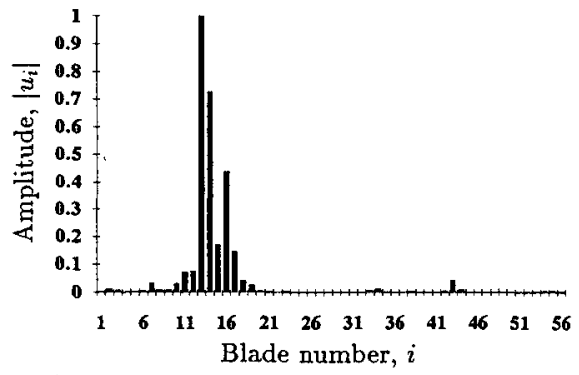

a) Nominal air density

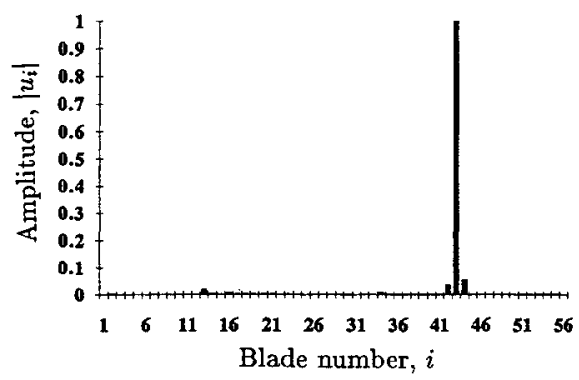

b) Air density one-fourth the nominal value

Fig. 9 Amplitude pattern of the highest-frequency mode $\lambda_{H}$ for mistuned assemblies. The mistuning strength is $\epsilon=4.76 \%$. Observe the much stronger localization featured by the assembly with the lower air density.

4) Equation (23) shows that the degree of localization of a mode depends only on the ratio of aerodynamic coupling to structural mistuning. Thus, localization increases as interblade coupling decreases or as mistuning increases. Finally, secondorder mode shape perturbations could be calculated but may not reveal new qualitative features.

\section{Results and Discussion}

The aeroelastic eigenvalue problem is solved for a tuned assembly and for several mistuning strengths, with the system parameters given in Sec. II. The mistuning values are obtained from a random number generator with a uniform probability distribution. We present typical results that demonstrate the extreme sensitivity of the blade assembly dynamics to mistuning and that confirm the general trends predicted by our perturbation analyses.

\section{A. Loss of Eigenstructure}

Figure 5 displays the root locus of the 56 aeroelastic eigenvalues in the complex plane for various mistuning values. The real part of the eigenvalues is plotted against the imaginary part. Note the regular pattern of the root locus of the perfectly tuned assembly in Fig. 5a, which is characteristic of the existence of constant interblade phase angle modes. Also note that all the eigenvalues have positive (although small) imaginary parts, which ensures stability in all modes. Finally, observe that all eigenvalues have real parts clustered in a relatively narrow interval (all frequencies of oscillation are within $5 \%$ of the assumed frequency). This is characteristic of weakly coupled systems, which feature closely spaced eigenvalues. Here the interblade coupling is aerodynamic and is thus weak, leading to a narrow band of frequencies for the tuned system.

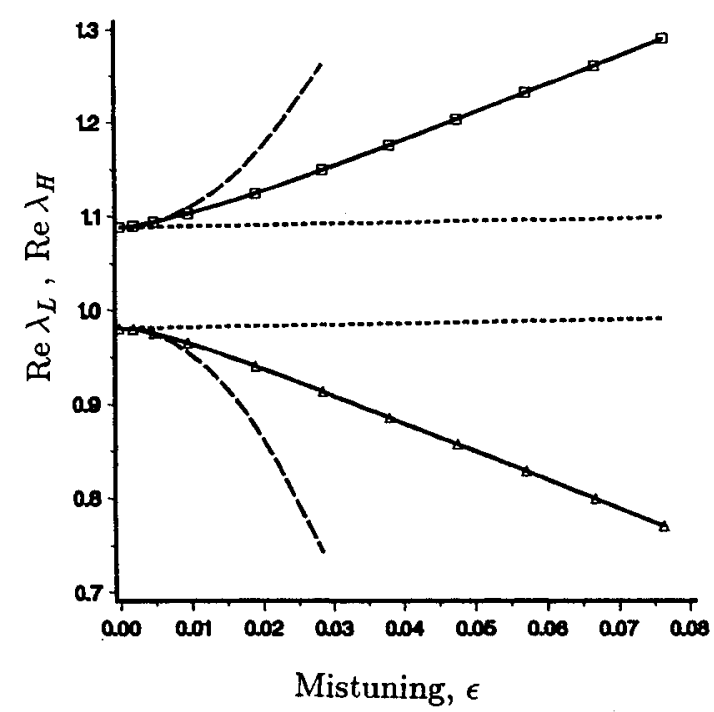

Fig. 10 Loci of the real parts (frequency) of the lowest- and highestfrequency eigenvalues vs mistuning, by "exact" numerical solution method (-), first-order classical perturbation method (- - ), and second-order classical perturbation method (- - -).

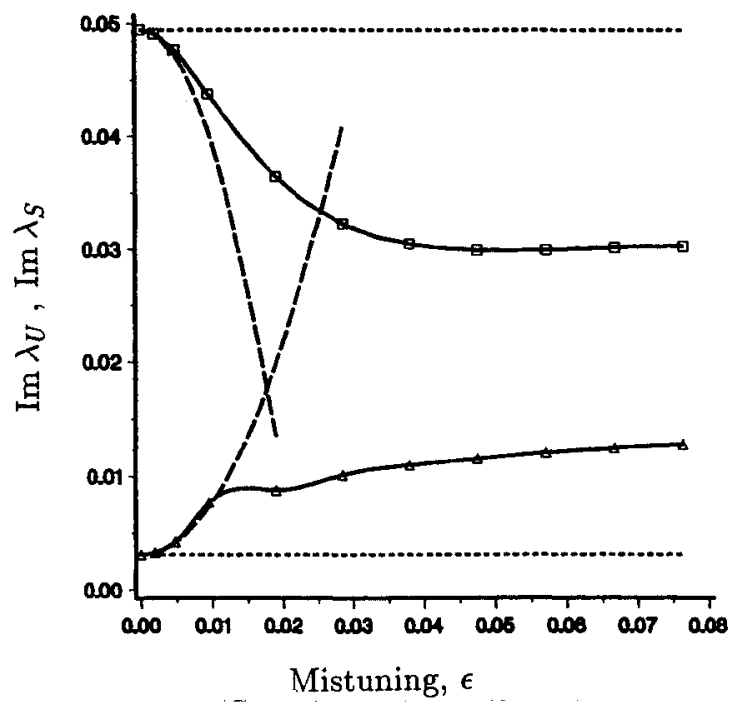

Fig. 11 Loci of the imaginary parts (damping) of the least and most stable eigenvalues vs mistuning, by "exact" numerical solution method $(-)$, first-order classical perturbation method $(--)$, and second-order classical perturbation method (- - -). 
Figures 5b-5d depict the root locus of mistuned assemblies. As mistuning increases we observe that the regularity of the root locus is gradually lost, and for small mistuning $\epsilon \geq 1.9 \%$ the locus consists of a constellation of eigenvalues with little discernible pattern. We refer to this phenomenon as loss of eigenstructure. It is yet another illustration of the extreme sensitivity of the eigensolution to mistuning. Although the mistuned eigenvalues are apparently randomly scattered, we observe two trends. 1) Mistuning results in the widening of the range of the real parts of the eigenvalues; i.e., the natural frequencies move apart when mistuning is introduced. This is consistent with the veering away of the lowest and highest frequencies shown in Fig. 3. 2) The imaginary parts of the eigenvalues (corresponding to aerodynamic damping) move closer together as mistuning increases. In particular, the least stable eigenvalue becomes more stable. This narrowing of the root locus along the imaginary direction confirms the veering toward of the least and most stable imaginary parts of the eigenvalues depicted in Fig. 4.

\section{B. Localization of Aeroelastic Modes}

Figure 6 displays the eigenvector corresponding to the lowest-frequency eigenvalue for various mistuning strengths. Both the amplitude pattern and the interblade phase angle pattern of the mode shapes are depicted. As expected, the mode shape of the tuned system features identical amplitudes for all blades and a constant interblade phase angle. When mistuning increases, the mode shape is altered fundamentally: The whole assembly ceases to participate in the motion and the vibration becomes confined to a few of the blades, with the others vibrating with negligible amplitudes. This indicates the occurrence of the phenomenon of localization of the aeroelastic mode shapes. Note that the constant interblade phase angle of the tuned system is lost when localization occurs, and no pattern emerges for the phase angles of the mistuned system's mode. Also note that the transition from extended to localized modes is very rapid in Fig. 6: Substantial localization already occurs for the very small mistuning $\epsilon=0.19 \%$, which is unavoidable in practice. Localization becomes severe as mistuning increases to $\epsilon=0.47 \%$. Although a single mode is displayed in Fig. 6, our results show that all the mode shapes of the mistuned assembly become localized.

The discussion in Sec. V.B suggests that the modes with the lowest and highest frequency are most sensitive to mistuning and thus may be the first to become localized. To confirm this

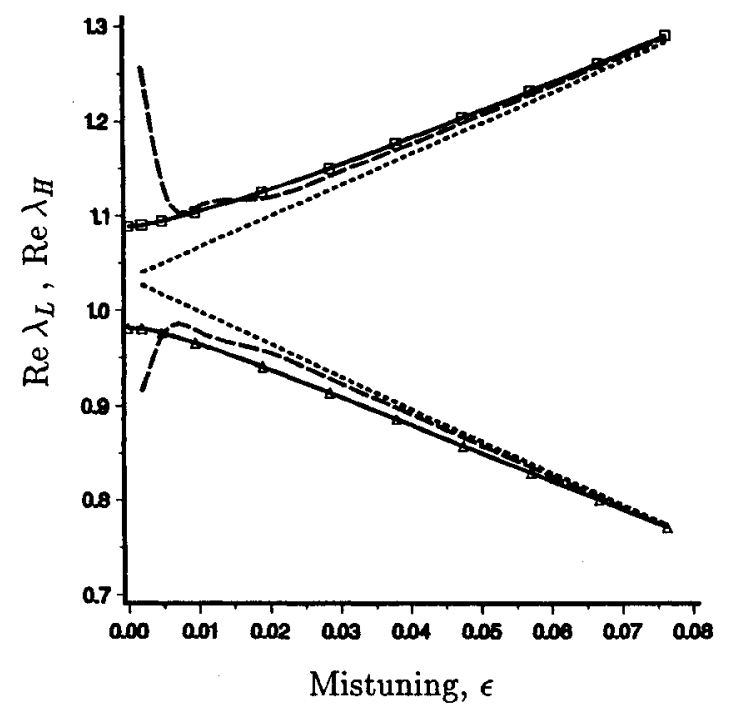

Fig. 12 Loci of the real parts (frequency) of the lowest- and highestfrequency eigenvalues vs mistuning, by "exact" numerical solution method (-), first-order modified perturbation method (- - -), and second-order modified perturbation method (- - ).

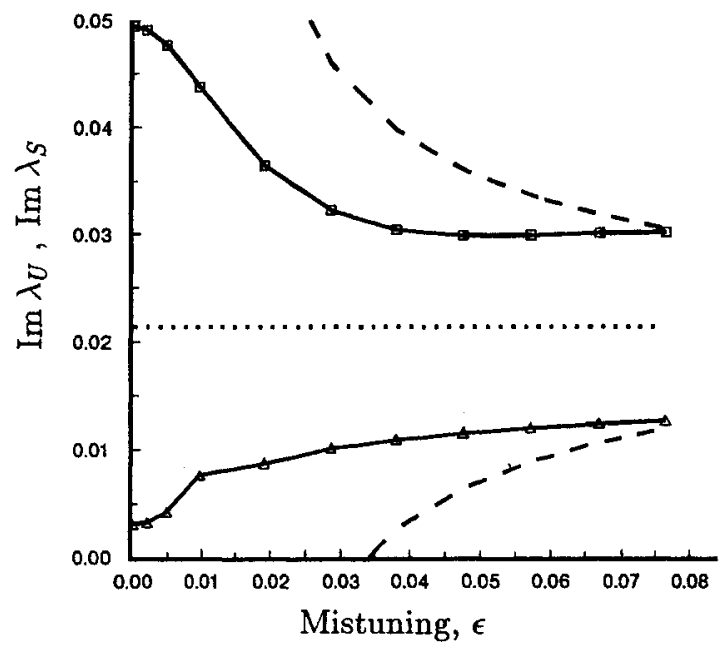

Fig. 13 Loci of the imaginary parts (damping) of the least and most stable eigenvalues vs mistuning, by "exact" numerical solution method ( - ), first-order modified perturbation method (- - -), and second-order modified perturbation method (- - -).

conjecture, Fig. 7 displays the amplitude patterns of four aeroelastic mode shapes for a given mistuning strength: two modes at the extremes of the frequency cluster, the lowest and highest frequency modes, and two modes near the middle of the frequency cluster, the least and most stable modes. Indeed, observe that the modes with the extreme frequencies are substantially more localized than those near the middle of the frequency band. This confirms our interpretation of Eq. (14). Although we do not show it here, we also observed that the four modes in Fig. 7 become more localized as mistuning increases, and achieve nearly the same degree of localization.

Another finding in Sec. V.B is that the sensitivity to mistuning increases with the number of blades. To illustrate this, Fig. 8 displays the amplitude patterns of the highest-frequency modes for mistuned assemblies made of 28 and 56 blades, where the number of blades was varied by keeping the blade geometry the same. We note that, although the mistuning standard deviation is the same for both assemblies, the mode of the 56-blade assembly is much more strongly localized than that of the 28-blade assembly. This confirms our finding that the degree of localization increases with the number of blades and means that mistuning has a greater impact on the dynamics of rotors with many blades such as turbines.

In Sec. $V$ we showed by perturbation methods that both the sensitivity to mistuning and the degree of localization increase as the interblade coupling decreases. In our model we can vary the unsteady aerodynamic coupling forces simply by adjusting the air density: A decrease in air density results in a decrease of all the elements of $\mathbf{A}$ by the same factor. Figure 9 displays mode shapes of assemblies with identical mistuning strength but different air densities. Observe that the modes become more strongly localized as the air density, and thus the interblade coupling, decreases.

\section{Comparison of Perturbation and Numerical Results}

Here we verify the validity of the perturbation results derived in Sec. V by comparing them with "exact" numerical results. Figure 10 depicts the loci of the real parts of the lowest- and highest-frequency eigenvalues vs mistuning. Both the first- and second-order classical perturbation results [Eqs. (11) and (14)] and the numerical solution of the aeroelastic eigenvalue problem (1) are shown. (The perturbation results were obtained by perturbing directly the lowest- and highestfrequency eigenvalues of the tuned system, not by sorting them at each mistuning level.) As discussed in Sec. V.A, we observe that the first-order eigenvalue perturbation does not capture the system's high sensitivity. The second-order perturbation solution provides the parabola tangent to the exact 
solution. It predicts the veering away of the loci and thus the high sensitivity to mistuning. However, this approximation is valid for very small mistuning only; for $\epsilon>0.5 \%$ it grossly overpredicts the exact solution.

Figure 11 displays the loci of the imaginary parts of the least and most stable eigenvalues, obtained by first- and second-order classical perturbations [Eqs. (11) and (15), applied directly to the least and most stable tuned eigenvalues] and by direct solution of Eq. (1). Again, only the second-order eigenvalue perturbation predicts the veering toward of the loci. Neither perturbation result approximates the exact solution adequately, except for very small mistuning $(\epsilon<0.5 \%)$.

The exact eigenvalues are compared with the first- and second-order modified perturbation results [Eqs. (18) and (21)] in Figs. 12 and 13. From the loci of the real parts in Fig. 12, we note that the modified perturbation method approximates the exact solution remarkably well away from the veering region. This means that the treatment of the small aerodynamic coupling as a perturbation in Sec. V.C is a valid methodology and that for small (but not too small) mistuning the modes have a localized character. As expected, the modified perturbation approximation deteriorates for very small mistuning, i.e., in the veering region. In this region the classical perturbation method must be used (see Figs. 10 and 11). The mistuning range where neither perturbation scheme gives accurate results defines the transition from constant interblade phase angle modes to localized modes.

In Fig. 13, which is for the imaginary parts of the least and most stable eigenvalues, the modified perturbation results agree relatively well with the exact solution. However, for a given mistuning level, the agreement in Fig. 13 is not as good as that observed for the frequencies in Fig. 12. Moreover, it should be noted that we had difficulties making sure that the perturbation and exact solutions displayed in Fig. 13 correspond to the same eigenvalues.

\section{Concluding Remarks}

The primary findings of our study consist of the following:

1) The sensitivity to mistuning is governed by the interblade coupling strength. Weak aerodynamic (or, for that matter, structural) coupling between blades results in high sensitivity to mistuning and qualitative alterations of the blade assembly's dynamics.

2) The root locus of the aeroelastic eigenvalues (frequency vs damping) loses the regular pattern that characterizes the tuned system to become apparently randomly scattered for small mistuning.

3) When plotted against mistuning strength, the real parts of the eigenvalues (the frequencies) veer away from each other with high curvature, whereas the imaginary parts of the eigenvalues (the damping values) veer toward each other abruptly.

4) The constant interblade phase angle aeroelastic mode shapes of the tuned assembly are severely altered when mistuning is present. The mistuned modes are not extended as in the tuned case, but each mode is strongly localized to a few blades of the assembly, and no pattern can be discerned for the interblade phase angles.

\section{Appendix A: Properties of Circulant Matrices}

Circulant matrices arise in the study of systems with perfect cyclic symmetry. The book by Davis ${ }^{17}$ contains a nice account of their properties. Here, we briefly state those properties that are used in the present work.

The general form of a square circulant matrix is

$$
\begin{aligned}
\mathbf{C} & =\operatorname{circ}\left(c_{1}, c_{2}, \ldots, c_{N}\right) \\
& =\left[\begin{array}{cccc}
c_{1} & c_{2} & \cdots & c_{N} \\
c_{N} & c_{1} & \cdots & c_{N-1} \\
\vdots & \vdots & \ddots & \vdots \\
c_{2} & c_{3} & \cdots & c_{1}
\end{array}\right], \quad c_{i} \in \mathcal{C}
\end{aligned}
$$

We note that a circulant matrix has only $N$ distinct elements. When several component modes are used to model a blade, the elements $c_{i}$ are themselves matrices and $\mathbf{C}$ is said to be block circulant.

Circulant matrices of order $N$ possess $N$ independent eigenvectors. Furthermore, all circulant matrices share the same set of eigenvectors:

$$
\begin{gathered}
\boldsymbol{e}_{j}=\frac{1}{\sqrt{N}}\left[1, e^{\frac{2 \pi i(j-1)}{N}}, e^{\frac{2 \pi i 2(j-1)}{N}}, \ldots, e^{\frac{2 \pi i(N-1)(j-1)}{N}}\right]^{T} \\
j=1, \ldots, N
\end{gathered}
$$

where the eigenvectors have been normalized such that $\left\|e_{j}\right\|=1$. An important property of the eigenvectors is that $\overline{\boldsymbol{e}}_{j}=\boldsymbol{e}_{N-j+2}$, for $j=2, \ldots, N$; thus, most eigenvectors occur in complex conjugate pairs, although $e_{1}$ is real and for $N$ even $\boldsymbol{e}_{N / 2+1}$ is real.

The eigenvalues of a circulant matrix can be written in closed form as

$\lambda_{j}=\sum_{j=1}^{N} c_{k} \exp \{[2 \pi i(j-1)(k-1)] / N\}, j=1, \ldots, N$

We can arrange the eigenvectors of a circulant matrix in an $N \times N$ modal matrix, as follows:

$$
\mathbf{E}=\left[e_{1}, \ldots, e_{j}, \ldots, e_{N}\right]
$$

The modal matrix $\mathbf{E}$ is commonly referred to as the Fourier matrix. It is a unitary matrix, i.e., $\mathbf{E}^{-1}=\mathbf{E}^{*}$. The modal matrix $\mathbf{E}$ diagonalizes any circulant matrix $\mathbf{C}$ through the similarity (and unitary) transformation:

$$
\mathbf{E}^{*} \mathbf{C E}=\operatorname{diag}\left(\lambda_{1}, \ldots, \lambda_{j}, \ldots, \lambda_{N}\right)
$$

where the eigenvalues of the diagonal matrix are given by Eq. (A3).

\section{Appendix B: Eigensolution Perturbations}

Consider the following eigenvalue problem:

$$
\left\{\mathbf{P}_{o}+\delta \mathbf{P}\right\} u_{j}=\lambda_{j} \mathbf{Q} u_{j}, \quad j=1, \ldots, N
$$

where $\mathbf{P}_{o}, \delta \mathbf{P}$, and $\mathbf{Q}$ are $N \times N$ complex matrices and $\left(\lambda_{j}, \boldsymbol{u}_{j}\right)$ an eigensolution. We denote the eigensolution of the unperturbed eigenvalue problem (for $\delta \mathbf{P} \equiv \mathbf{0})$ by $\left(\lambda_{o j}, \boldsymbol{u}_{o j}\right)$, $j=1, \ldots, N$. Furthermore, we introduce the transposed unperturbed eigenvalue problem as

$$
\mathbf{P}_{o}^{T} \boldsymbol{v}_{o j}=\mu_{o j} \mathbf{Q}^{T} \boldsymbol{v}_{o j} \quad j=1, \ldots, N
$$

Note that Eq. (B2) is not the adjoint eigenvalue problem. One can easily show that $\mu_{o j}=\lambda_{o j}$ and that the two sets of eigenvectors of the unperturbed problems are biorthogonal:

$$
\left\{\begin{array}{l}
\boldsymbol{v}_{o j}^{T} \mathbf{Q} \boldsymbol{u}_{o k}=0 \\
\boldsymbol{v}_{o j}^{T} \mathbf{P} \boldsymbol{u}_{o k}=0
\end{array} \quad j \neq k\right.
$$

Next, we expand the eigensolution of the perturbed problem, Eq. (B1), to the second order as

$$
\left\{\begin{array}{l}
\lambda_{j}=\lambda_{o j}+\delta \lambda_{j}+\delta^{2} \lambda_{j} \\
\boldsymbol{u}_{j}=\boldsymbol{u}_{o j}+\delta \boldsymbol{u}_{j}+\delta^{2} \boldsymbol{u}_{j}
\end{array} \quad j=1, \ldots, N\right.
$$

The eigenvalue perturbations can be shown to be

$$
\begin{array}{r}
\delta \lambda_{j}=\frac{v_{o j}^{T} \delta \mathbf{P} \boldsymbol{u}_{o j}}{\boldsymbol{v}_{o j}^{T} \mathbf{Q} \boldsymbol{u}_{o j}} \quad j=1, \ldots, N \\
\delta^{2} \lambda_{j}=\sum_{\substack{k=1 \\
k \neq j}}^{N} \frac{1}{\lambda_{o j}-\lambda_{o k}} \frac{\left(v_{o k}^{T} \delta \mathbf{P} u_{o j}\right)\left(v_{o j}^{T} \delta \mathbf{P} \boldsymbol{u}_{o k}\right)}{\left(v_{o k}^{T} \mathbf{Q} u_{o k}\right)\left(v_{o j}^{T} \mathbf{Q} u_{o j}\right)} \\
j=1, \ldots, N
\end{array}
$$


The first-order eigenvector perturbation is

$$
\delta \boldsymbol{u}_{j}=\sum_{\substack{k=1 \\ k \neq j}}^{N} \frac{1}{\lambda_{o j}-\lambda_{o k}} \frac{\boldsymbol{v}_{o k}^{T} \delta \mathbf{P} u_{o j}}{\boldsymbol{v}_{o k}^{T} \mathbf{Q} u_{o k}} \boldsymbol{u}_{o k} \quad j=1, \ldots, N
$$

We do not give $\delta^{2} u_{j}$ since we do not make use of it in the paper. Reference 16 describes the general approach to prove Eqs. (B5-B7).

For the aeroelastic eigenvalue problem, Eq. (1), we have $\mu_{o j}=\lambda_{o j}, \boldsymbol{u}_{o j}=\boldsymbol{e}_{j}$, and $\boldsymbol{v}_{o j}=\overline{\boldsymbol{e}}_{j}$.

\section{Acknowledgments}

This work was supported by the Institute for Computational Mechanics in Propulsion at NASA Lewis Research Center and by NASA Lewis Grant NAG3-1163, Structural Dynamics Branch. The development of the ideas contained in this paper was aided by discussions with Todd Smith of Sverdrup Corporation. The authors also thank Gisli Ottarsson for his contributions to the sections on circulant matrices and tuned system dynamics.

\section{References}

${ }^{1}$ Lane, F., "System Mode Shapes in the Flutter of Compressor Blade Rows," Journal of Aeronautical Sciences, Vol. 13, 1956, pp. 54-66.

${ }^{2}$ Crawley, E. F., "Aeroelastic Formulation for Tuned and Mistuned Rotors," AGARD Manual AG-298 on Aeroelasticity in AxialFlow Turbomachines, Vol. 2, 1988, Chap. 19.

${ }^{3}$ Srinivasan, A. V., "Vibrations of Bladed-Disk Assemblies-A Selected Survey," Journal of Vibration, Acoustics, Stress, and Reliability in Design, Vol. 106, No. 2, 1984, pp. 165-168.

${ }^{4}$ Kielb, R. E., and Kaza, K. R. V., "Aeroelastic Characteristics of a Cascade of Mistuned Blades in Subsonic and Supersonic Flows," Journal of Vibration, Acoustics, Stress, and Reliability in Design, Vol. 105, 1983, pp. 425-433.

${ }^{5}$ Kielb, R. E., and Kaza, K. R. V., "'Effects of Structural Coupling on Mistuned Cascade Flutter and Response," Journal of Engineering for Gas Turbines and Power, Vol. 106, No. 1, 1984, pp. 17-24.

"Bendiksen, O. O., "Recent Developments in Flutter Suppression Techniques for Turbomachinery Rotors," Journal of Propulsion and Power, Vol. 4, No. 2, 1988, pp. 164-171.

${ }^{7}$ Bendiksen, O. O., "Flutter of Mistuned Turbomachinery Rotors," Journal of Engineering for Gas Turbines and Power, Vol. 106, 1984, pp. 25-33.

${ }^{8}$ El-Bayoumy, L. E., and Srinivasan, A. V., "Influence of Mistuning on Rotor-Blade Vibrations,"' AIAA Journal, Vol. 13, No. 4, 1975, pp. 460-464.

${ }^{9}$ Ewins, D. J., "Vibration Characteristics of Bladed Disc Assemblies," Journal of Mechanical Engineering Science, Vol. 15, No. 3, 1973, pp. 165-186.

${ }_{10}$ Valero, N. A., and Bendiksen, O. O., "Vibration Characteristics of Mistuned Shrouded Blade Assemblies," ASME Journal of Engineering for Gas Turbines and Power, Vol. 108, No. 2, 1986, pp. 293-299.

${ }^{11}$ Wei, S. T., and Pierre, C., "Localization Phenomena in Mistuned Assemblies with Cyclic Symmetry. Part I: Free Vibrations," Journal of Vibration, Acoustics, Stress, and Reliability in Design, Vol. 110, No. 4, 1988, pp. 429-438.

${ }^{12}$ Wei, S. T., and Pierre, C., "Localization Phenomena in Mistuned Assemblies with Cyclic Symmetry. Part II: Forced Vibrations," Journal of Vibration, Acoustics, Stress, and Reliability in Design, Vol. 110, No. 4, 1988, pp. 439-449.

${ }^{13}$ Kaza, K. R. V., and Kielb, R. E., "Flutter of Turbofan Rotors with Mistuned Blades," AIAA Journal, Vol. 22, No. 11, 1984, pp. $1618-1625$.

${ }^{14}$ Rao, B. M., and Jones, W. P., "Unsteady Airloads on a Cascade of Staggered Blades in Subsonic Flow,' 46th Propulsion Energetics Review Meeting, Monterey, CA, AGARD-CP-177, 1975, Paper 32.

${ }^{15}$ Adamczyk, J. J., and Goldstein, M. E., "Unsteady Flow in Supersonic Cascade with Subsonic Leading-Edge Locus," AIAA Journal, Vol. 16, No. 12, 1973, pp. 1248-1254.

${ }^{16}$ Courant, R., and Hilbert, D., Methods of Mathematical Physics, Vol. I, Interscience, New York, 1953.

${ }^{17}$ Davis, P. J., Circulant Matrices, Wiley-Interscience, New York, 1979. 